\title{
Two-dimensional multi-component photometric decomposition of CALIFA galaxies *
}

\author{
J. Méndez-Abreu ${ }^{1}$, T. Ruiz-Lara ${ }^{2,3}$, L. Sánchez-Menguiano ${ }^{2,4}$, A. de Lorenzo-Cáceres ${ }^{1,2}$, L. Costantin ${ }^{5}$, \\ C. Catalán-Torrecilla ${ }^{6}$, E. Florido ${ }^{2,3}$, J. A. L. Aguerri ${ }^{7,8}$, J. Bland-Hawthorn ${ }^{9}$, E. M. Corsini ${ }^{5,10}$, R. J. Dettmar ${ }^{11}$, \\ L. Galbany ${ }^{12,13}$, R. García-Benito ${ }^{4}$, R. A. Marino ${ }^{14}$, I. Márquez ${ }^{4}$, R. A. Ortega-Minakata ${ }^{15}$, P. Papaderos ${ }^{16}$, \\ S. F. Sánchez ${ }^{17}$, P. Sánchez-Blazquez ${ }^{18,19}$, K. Spekkens ${ }^{20}$, G. van de Ven ${ }^{21}$, V. Wild ${ }^{1}$, and B. Ziegler ${ }^{22}$
}

${ }^{1}$ School of Physics and Astronomy, University of St. Andrews, SUPA, North Haugh, KY16 9SS, St. Andrews, UK e-mail: jma20@st-andrews.ac.uk

2 Departamento de Física Teórica y del Cosmos, Universidad de Granada, Campus de Fuentenueva, 18071 Granada, Spain

3 Instituto Carlos I de Física Teórica y Computacional, Universidad de Granada, 18071 Granada, Spain

${ }^{4}$ Instituto de Astrofísica de Andalucía (CSIC), Glorieta de la Astronomía s/n, 3004, 18080 Granada, Spain

5 Dipartimento di Fisica e Astronomia “G. Galilei”, Università di Padova, vicolo dell'Osservatorio 3, 35122 Padova, Italy

6 Departamento de Astrofísica y CC. de la Atmósfera, Universidad Complutense de Madrid, 28040 Madrid, Spain

7 Instituto de Astrofísica de Canarias, Calle Vía Láctea s/n, 38205 La Laguna, Tenerife, Spain

8 Departamento de Astrofísica, Universidad de La Laguna, 38200 La Laguna, Tenerife, Spain

9 Sydney Institute for Astronomy, School of Physics A28, University of Sydney, NSW 2006, Australia

10 INAF-Osservatorio Astronomico di Padova, vicolo dell'Osservatorio 5, 35122 Padova, Italy

11 Astronomisches Institut, Ruhr-Universität Bochum, Universitätsstr. 150, 44801 Bochum, Germany

12 Pittsburgh Particle Physics, Astrophysics, and Cosmology Center (PITT PACC), 3941 O'Hara Street Pittsburgh, PA 15260, USA

13 Physics and Astronomy Department, University of Pittsburgh, Pittsburgh, PA 15260, USA

14 ETH Zürich, Institute for Astronomy, Wolfgang-Pauli-Str. 27, 8093 Zürich, Switzerland

15 Observatório do Valongo, Universidade Federal do Rio de Janeiro, Ladeira do Pedro Antônio 43, Saúde, Rio de Janeiro, RJ 20080-090, Brazil

16 Instituto de Astrofísica e Ciências do Espaço, Universidade do Porto, Centro de Astrofísica da Universidade do Porto, Rua das Estrelas, 4150-762 Porto, Portugal

17 Instituto de Astronomía, Universidad Nacional Autónoma de México, A.P. 70-264, 04510 México D.F., Mexico

18 Departamento de Física Teórica, Universidad Autónoma de Madrid, 28049 Cantoblanco, Spain

19 Instituto de Astrofśica, Pontificia Universidad Católica de Chile, Av. Vicuña Mackenna 4860, 782-0436 Macul, Santiago, Chile

20 Department of Physics, Royal Military College of Canada, PO Box 17000, Station Forces, Kingston, Ontario K7K 7B4, Canada

21 Max-Planck-Institut für Astronomie, Königstuhl 17, 69117 Heidelberg, Germany

${ }^{22}$ University of Vienna, Department of Astrophysics, Türkenschanzstrasse 17, 1180 Vienna, Austria

Received 13 August 2016 / Accepted 10 October 2016

\begin{abstract}
We present a two-dimensional multi-component photometric decomposition of 404 galaxies from the Calar Alto Legacy Integral Field Area data release 3 (CALIFA-DR3). They represent all possible galaxies with no clear signs of interaction and not strongly inclined in the final CALIFA data release. Galaxies are modelled in the $g, r$, and $i$ Sloan Digital Sky Survey (SDSS) images including, when appropriate, a nuclear point source, bulge, bar, and an exponential or broken disc component. We use a human-supervised approach to determine the optimal number of structures to be included in the fit. The dataset, including the photometric parameters of the CALIFA sample, is released together with statistical errors and a visual analysis of the quality of each fit. The analysis of the photometric components reveals a clear segregation of the structural composition of galaxies with stellar mass. At high masses $\left(\log \left(M_{\star} / M_{\odot}\right)>11\right)$, the galaxy population is dominated by galaxies modelled with a single Sérsic or a bulge+disc with a bulge-tototal $(\mathrm{B} / \mathrm{T})$ luminosity ratio $\mathrm{B} / \mathrm{T}>0.2$. At intermediate masses $\left(9.5<\log \left(M_{\star} / M_{\odot}\right)<11\right)$, galaxies described with bulge+disc but $\mathrm{B} / \mathrm{T}<0.2$ are preponderant, whereas, at the low mass end $\left(\log \left(M_{\star} / M_{\odot}\right)<9.5\right)$, the prevailing population is constituted by galaxies modelled with either pure discs or nuclear point sources+discs (i.e., no discernible bulge). We obtain that $57 \%$ of the volume corrected sample of disc galaxies in the CALIFA sample host a bar. This bar fraction shows a significant drop with increasing galaxy mass in the range $9.5<\log \left(M_{\star} / M_{\odot}\right)<11.5$. The analyses of the extended multi-component radial profile result in a volume-corrected distribution of $62 \%, 28 \%$, and $10 \%$ for the so-called Type I (pure exponential), Type II (down-bending), and Type III (up-bending) disc profiles, respectively. These fractions are in discordance with previous findings. We argue that the different methodologies used to detect the breaks are the main cause for these differences.
\end{abstract}

Key words. galaxies: bulges - galaxies: evolution - galaxies: formation - galaxies: stellar content - galaxies: structure galaxies: photometry

\footnotetext{
$\star$ The catalog of fitted parameters is only available at the CDS via anonymous ftp to cdsarc.u-strasbg. fr (130.79.128.5) or via http://cdsarc.u-strasbg.fr/viz-bin/qcat?J/A+A/598/A32
}

\section{Introduction}

The constant development of the morphological classification schemes, from the Hubble tuning fork diagram (Hubble 1926) 
to the more sophisticated Comprehensive de Vaucouleurs revised Hubble-Sandage (CVRHS; Buta et al. 2015) catalogue, illustrate the morphological complexity of galaxy systems. Even apparently simple systems like ellipticals can host a wealth of other structures such as outer shells or nuclear cores (Malin \& Carter 1980; Morelli et al. 2004). The properties of the different stellar structures that make up galaxies (e.g., bulges, bars, and discs) are the direct result of their formation and evolution. Therefore, the quantification of the properties of galaxies and their distinct stellar structures is a fundamental step towards understanding how galaxies form and evolve.

Historically, since the early era of photographic plates, one of the key methods for studying the projected luminosity density, or surface brightness (SB), of galaxies was to use parametric laws to model their different components (Freeman 1970; Kormendy 1977). In the early 80s, the pioneering photometric decomposition methods were based on modelling the onedimensional (1D) SB profiles of galaxies as the sum of separate components (e.g. bulge+disc; Boroson 1981; Send 1982; Hickson et al. 1982). With the advent of charge couple device (CCD) cameras, these first attempts to understand the stellar structures that shape a galaxy were improved upon in the following decades by hybrid methods fitting not only the SB but also the galaxy ellipticity profiles (Trujillo et al. 2001b; Aguerri \& Trujillo 2002). The first pixel-by-pixel fitting of galaxy images with two-dimensional (2D) SB models was done by Byun \& Freeman (1995). Since then, several codes have been developed to perform 2D bulge+disc photometric decompositions such as GIM2D (Simard 1998), but most recent algorithms allow for a 2D multi-component photometric decomposition (e.g. GALFIT, Peng et al. 2002, BUDDA, de Souza et al. 2004, GASP2D, Méndez-Abreu et al. 2008, IMFIT, Erwin 2015).

The necessity for a multi-component approach to the photometric decomposition of galaxies has been demonstrated in several works (Prieto et al. 2001; Gadotti 2008; Salo et al. 2015). In particular, the inclusion of the bar component has been proved to be critical in order to recover accurate bulge parameters (e.g. Aguerri et al. 2005; Laurikainen et al. 2005). Several studies have shown that both the Sérsic index $(n)$ and the bulgeto-total luminosity ratio $(\mathrm{B} / \mathrm{T})$ can be artificially increased if the bar is not properly accounted for in the fit (Gadotti 2009; Weinzirl et al. 2009; Méndez-Abreu et al. 2014). In addition, recent advances in observational techniques have allowed for detailed analysis of the light distribution in the outermost regions of galaxies. Despite the classical view of galaxies hosting pure exponential discs being confirmed with ultra deep observations (e.g. Bland-Hawthorn et al. 2005), a wide variety of outer profiles deviating from a pure exponential have also been reported (Erwin et al. 2005; Pohlen \& Trujillo 2006). Therefore, since the origin of breaks in disc profiles is still debated, a multicomponent approach taking into account broken profiles is of vital importance for understanding disc formation and evolution processes (Marino et al. 2016; Ruiz-Lara et al. 2016).

Despite their limitations, two-component (bulge+disc) photometric decompositions are still the common procedure when dealing with large surveys at low and high redshift (Allen et al. 2006; Simard et al. 2011; Lackner \& Gunn 2012; Häußler et al. 2013). This is mainly because current methodologies to find the best fit model to the galaxy images using two-component models are relatively easy to automatise. However, when more structures are added to the fitting process, they become more degenerate and human supervision is usually needed. A number of studies have attempted to produce multi-component photometric decompositions of samples with several hundreds of galaxies. Recently, Salo et al. (2015) performed the largest multi-component decomposition to date, analysing 2352 galaxies from the Spitzer Survey of Stellar Structure in Galaxies $\left(S^{4} \mathrm{G}\right.$, Sheth et al. 2010).

Here, we present the $2 \mathrm{D}$ multi-component photometric decomposition of 404 galaxies drawn from the final data release of the Calar Alto Legacy Integral Field Area survey (CALIFA, Sánchez et al. 2012). The galaxy sample represents all galaxies in the CALIFA survey that are suitable for our photometric analysis, that is, they are not in interaction with other galaxies and are not heavily inclined. The CALIFA survey is an integral field spectroscopy (IFS) survey of 667 galaxies that provides spatially resolved information such as stellar and gas kinematics, stellar populations, and gas-phase physical properties over a large field of view (2-3 galaxy effective radii). The CALIFA data have significantly improved our understanding of the physical processes leading to the observed population of galaxies in the nearby Universe. The aim of this paper is to provide the CALIFA dataset with an accurate photometric characterisation of the multiple stellar structures shaping the CALIFA galaxies (bulges, bars, and discs). To this aim, we have used the homogeneous imaging provided by the Sloan Digital Sky Survey data release 7 (Abazajian et al. 2009, SDSS-DR7) for the whole CALIFA sample. Some of the information presented here has already been used within the survey collaboration to study the influence of bars in stellar population gradients (Sánchez-Blázquez et al. 2014), to address the stellar populations of different disc profiles (Ruiz-Lara et al. 2016), to understand gas abundance gradient in discs (Sánchez et al. 2014; Sánchez-Menguiano et al. 2016), and to analyse the morpho-kinematic properties of bulges in lenticular galaxies (Méndez-Abreu et al. 2016). This paper focuses on the technical aspects of the photometric decomposition and the incidence of the different galaxy structures in the CALIFA galaxy sample. The detailed photometric description of the galaxy structures presented in this paper opens a new set of possibilities to the wealth of 2D spatially resolved spectroscopic information provided by the CALIFA survey. The properties of the individual structures and their relation with other galaxy properties extracted from the CALIFA data will be explored in future papers.

The current paper is organised as follows: Sect. 2 describes the CALIFA data release 3 (DR3) and the final sample used in this study. Section 3 details the technical aspects of the 2D photometric decomposition analysis. Section 4 describes the fitting process and the main types of multi-component decomposition carried out in this paper. The incidence of the main stellar structures found in our sample are analysed in the context of the global properties of the galaxies. Section 5 presents a complete description of the uncertainties inherent to our analysis. The conclusions are given in Sect. 6. Throughout the paper we assume a flat cosmology with $\Omega_{\mathrm{m}}=0.3, \Omega_{\Lambda}=0.7$, and a Hubble constant $H_{0}=70 \mathrm{~km} \mathrm{~s}^{-1} \mathrm{Mpc}^{-1}$.

\section{The CALIFA DR3 and our sample selection}

The CALIFA final data release (DR3, Sánchez et al. 2016) comprises two different samples of galaxies: galaxies belonging to the CALIFA mother sample and galaxies that are considered the extension sample. The first group represents the natural expansion of the galaxies presented in the previous CALIFA DR1 (Husemann et al. 2013) and DR2 (García-Benito et al. 2015), and fully characterised in Walcher et al. (2014). The second group corresponds to a compendium of different sets of galaxies that were observed using the same setup as CALIFA, as part 

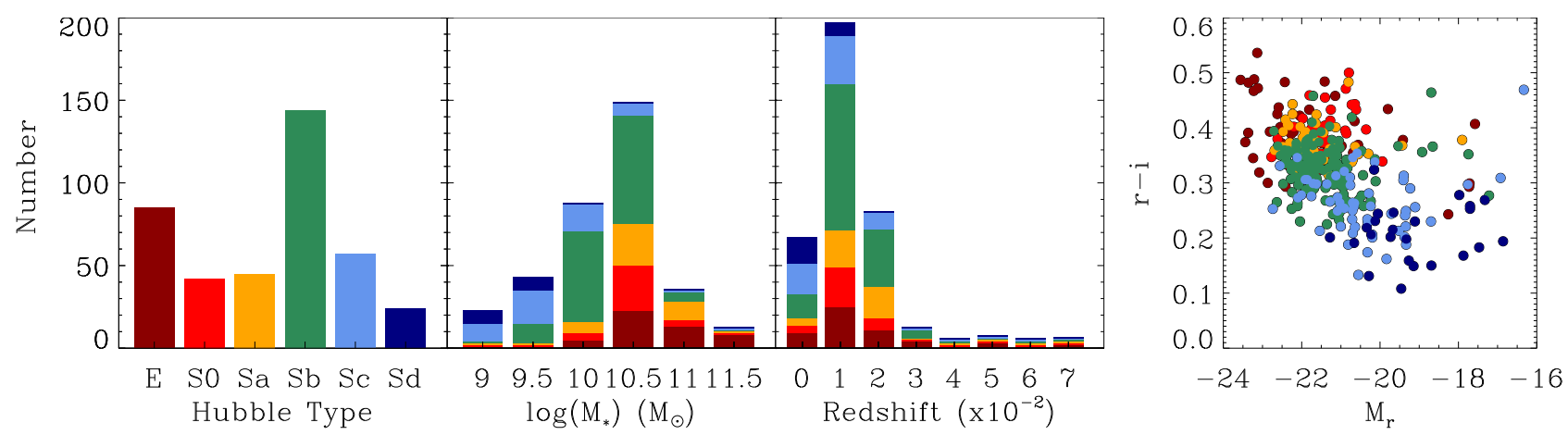

Fig. 1. (From left to right) Distribution of our galaxy sample in Hubble type, stellar mass, redshift, and on the $r-i$ vs. $r$-band colour magnitude relation. Colours in all panels represent the different galaxy Hubble types. The parameters for each galaxy were obtained from Walcher et al. (2014).

of ancillary science projects within the CALIFA collaboration. The final CALIFA DR3 comprises 667 galaxies.

From the final data release, and after performing a visual inspection of the SDSS images, we carry out a discard/exclusion process of those galaxies not suitable for our photometric study. First, we reject paired and interacting objects (57 galaxies) as well as those systems with a heavily distorted morphology (19 galaxies). Since we aim to provide an accurate description of the galaxy stellar structures using only symmetric models, galaxies with distorted features cannot be successfully modelled. Then, we check for the presence of bright stars contaminating our target galaxies and remove them from the analysis ( 5 galaxies). Finally, the identification and subsequent characterisation of structures in highly inclined galaxies is usually not possible due to projection effects, thus those galaxies close to edge-on $\left(i>70^{\circ}\right)$ were also removed from the sample (183 galaxies). This latter process was performed by a visual inspection of individual galaxies since we find that a typical disc axis ratio cut does not work for early-type edge-on galaxies with spherical stellar haloes. The final sample presented in this paper contains 404 galaxies. The distribution of their main global characteristics extracted from Walcher et al. (2014) is shown in Fig. 1.

The CALIFA mother sample presents the noticeable characteristic that its selection criteria are well understood. Therefore, although the final observed sample is not complete in volume, it is possible to reconstruct volume corrected sample properties. The complete procedure is described in Walcher et al. (2014) and assigns a volume correction to each individual galaxy that can be used to correct for the selection function. Figure 2 shows the luminosity function of the sample in this study, the CALIFA mother sample, and the final observed sample in the CALIFA DR3. It is worth noticing that volume corrections are not applicable to the extended sample due to their complicated sample selection. Thus, the luminosity function represented in Fig. 2 contains only those galaxies drawn from the CALIFA mother sample (297 galaxies). We find a good match among the luminosity functions of the three different samples, as well as for the SDSS luminosity function given by Blanton et al. (2003), within the completeness limits described in Walcher et al. (2014), that is, $-19>M_{r}>-23.1$. Our sample contains 285 galaxies within these limits.

\section{2D Photometric decomposition analysis: model functions and data reduction}

The structural parameters of the CALIFA sample were derived by applying a $2 \mathrm{D}$ photometric decomposition. The use of

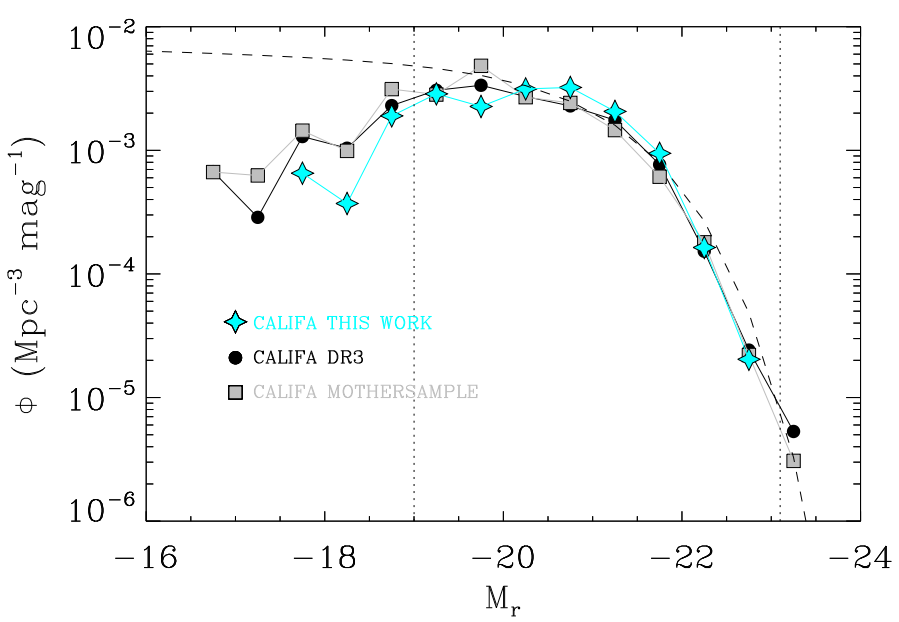

Fig. 2. Luminosity functions of the CALIFA mother sample (grey squares), final CALIFA observed sample in the DR3 (black dots), and the sample described in this paper (cyan stars). The dotted lines denote the mother sample completeness limits. The SDSS luminosity function of Blanton et al. (2003) is shown with a black dashed line.

algorithms fitting the 2D surface-brightness distributions (SBD) of galaxies has become standard practice in the analysis of galaxy photometric structures due to the advantages over simple $1 D$ analysis (see, Byun \& Freeman 1995; Erwin 2015). In this paper we apply the GASP2D code (Méndez-Abreu et al. 2008, 2014). GASP2D adopts a Leverberg-Marquard algorithm to fit the 2D SBD of galaxies with a versatile set of model components. This section presents the analytical functions chosen to describe each galaxy model as well as our pre-processing of the SDSS images to be used in the photometric decomposition.

\subsection{Analytical functions for the structural components}

The galaxy SBD is assumed to be the sum of multiple photometric structures depending on its specific morphology. GASP2D allows the simultaneous fitting of different galaxy structures: nuclear point source (NPS), bulge, bar, and disc (including breaks). Each of these components is built such that its corresponding isophotes are perfect ellipses centred on $\left(x_{0}, y_{0}\right)$ with constant position angle (PA) and constant ellipticity $(\epsilon=1-q)$, where $q$ is the minor-to-major axis ratio of the ellipse. The geometric parameters $(\mathrm{PA}, \epsilon)$ are independent for each component. The 
isophotal radius $r$ is then given by

$$
\begin{aligned}
r= & {\left[\left(-\left(x-x_{0}\right) \sin \mathrm{PA}+\left(y-y_{0}\right) \cos \mathrm{PA}\right)^{2}\right.} \\
& \left.+\left(-\left(x-x_{0}\right) \cos \mathrm{PA}-\left(y-y_{0}\right) \sin \mathrm{PA}\right)^{2} / q^{2}\right]^{1 / 2} .
\end{aligned}
$$

The SBD of the bulge component is parameterised with a Sérsic profile (Sérsic 1968), also known as the $r^{1 / n}$ law or generalised de Vaucouleurs law.

$I_{\mathrm{b}}\left(r_{\mathrm{b}}\right)=I_{\mathrm{e}} 10^{-b_{n}\left[\left(\frac{r_{\mathrm{b}}}{r_{\mathrm{e}}}\right)^{\frac{1}{n}}-1\right]}$,

where $r_{\mathrm{b}}$ is the radius measured in the Cartesian coordinates describing the reference system of the bulge in the plane of the sky. $r_{\mathrm{e}}, I_{\mathrm{e}}$, and $n$ are the effective (or half-light) radius, the surface brightness at $r_{\mathrm{e}}$, and the Sérsic index describing the curvature of the SBD, respectively, and $b_{n} \simeq 0.868 n-0.142$ (Caon et al. 1993).

The NPS is modelled using a Moffat function mimicking the point spread function (PSF) of the galaxy image. Several works have proven the importance of including a NPS to properly derive the bulge parameters when nuclear stellar clusters (NSC, Balcells et al. 2007) or active galactic nuclei (AGN, Benítez et al. 2013) are present. In this paper, the inclusion of a NPS does not intend to model an extra component (NSC, AGN) but rather to model an unresolved bulge with a size comparable to the image PSF. Therefore, the use of a NPS in the modelling of a galaxy rules out the simultaneous fitting with a bulge component (see Sect. 4.3.3 for further details). The NPS parameterisation is given by

$I_{\mathrm{NPS}}\left(r_{\mathrm{NPS}}\right)=I_{\mathrm{NPS}}\left(1+\left(\frac{r_{\mathrm{NPS}}}{\alpha}\right)^{2}\right)^{-\beta}$,

where the parameters $\alpha$ and $\beta$ define the profile shape and are related to the full width at half maximum (FHWM) such as $F W H M=2 \alpha \sqrt{2^{1 / \beta}-1}$.

The SBD of a galaxy disc is usually described with an exponential profile. However, nowadays it is commonly accepted that galaxy discs can be classified into three general categories (Erwin et al. 2005; Pohlen \& Trujillo 2006): (i) Type I profiles that follow a single exponential profile along the whole optical extent of the galaxies; (ii) Type II profiles that present a double exponential law with a down-bending beyond the so-called break radius; and (iii) Type III profiles that exhibit an up-bending in the outer parts of the discs. To account for these possibilities we adopt the following parameterisation:

$I_{\mathrm{d}}\left(r_{\mathrm{d}}\right)=I_{0}\left[\mathrm{e}^{\frac{-r_{\mathrm{d}}}{h}} \theta+\mathrm{e}^{\frac{-r_{\text {break }}\left(h_{\text {out }}-h\right)}{h_{\text {out }} h}} \mathrm{e}^{\frac{-r_{\mathrm{d}}}{h_{\text {out }}}}(1-\theta)\right]$,

where

$\theta=\left\{\begin{array}{lll}0 & \text { if } & r_{\mathrm{d}}>r_{\text {break }} \\ 1 & \text { if } & r_{\mathrm{d}}<r_{\text {break }}\end{array}\right.$

and $r_{\mathrm{d}}$ is the radius measured in the Cartesian coordinates describing the reference system of the disc. $I_{0}, h, h_{\text {out }}$, and $r_{\text {break }}$ are the central surface brightness, inner scale-length, outer scalelength, and break radius of the disc, respectively. A set of more elaborate functions to describe the break of exponential discs has been proposed in the literature (e.g. Peng et al. 2010; Erwin 2015). However, we opted for a simpler description in order to minimise the number of free parameters involved in the fitting process.
The projected surface density of a three-dimensional Ferrers ellipsoid (Ferrers 1877, see also Aguerri et al. 2009) is used to describe the SBD of the bar component;

$I_{\mathrm{bar}}\left(r_{\mathrm{bar}}\right)=I_{0, \mathrm{bar}}\left[1-\left(\frac{r_{\mathrm{bar}}}{a_{\mathrm{bar}}}\right)^{2}\right]^{n_{\mathrm{bar}}+0.5} ; \quad$ for $r_{\mathrm{bar}} \leq a_{\mathrm{bar}}$,

where $r_{\text {bar }}$ is the radius measured in the Cartesian coordinates describing the reference system of the bar. $I_{0, \text { bar }}, a_{\mathrm{bar}}$, and $n_{\mathrm{bar}}$ represent the central surface brightness, length, and shape parameter of the bar, respectively. Due to the high degree of degeneracy that the $n_{\text {bar }}$ parameter introduces during the fit, we decided to keep it as a fixed parameter during the fitting process. The default value used was $n_{\text {bar }}=2$ (see also Laurikainen et al. 2005). Two galaxies in the sample appeared to host a nuclear bar after a careful inspection of the 2D residuals and their SBD was also fitted using a Ferrers profile.

To derive the photometric parameters of the different structures present in a given galaxy, we iteratively fit a composite model made of a suitable combination of the previously described stellar components. The actual fitting for each galaxy is human supervised. This means that the final number of components included in the fit is based on the judgement of the codeuser. Usually, at least two different component combinations are tested for each galaxy before the best solution is found. The decision is based on the $2 \mathrm{D}$ distribution of the intensity residuals and the 1D surface brightness, ellipticity, and position angle radial profiles (see Sect. 4). Figure 3 shows an example of the plots used to choose the final number of stellar structures in UGC 11228. Upper panels show the 2D SBD for the galaxy, model, and residuals, and the lower panels represent the $1 \mathrm{D}$ radial profiles of the surface brightness, ellipticity and position angle. In this particular case, the best fit is achieved using a threecomponent model with a bulge, a bar, and a single exponential disc. Figure 3 clearly shows that a break exponential profile is not necessary to reproduce the SBD of UGC 11228, but the bar is indispensable to reproduce the bump in the $1 \mathrm{D}$ azimuthally averaged ellipticity and PA profiles.

An exception to the previous human supervised fitting procedure is the case of featureless early-type galaxies. If a given galaxy is visually classified as early-type (elliptical or S0/0a) and other structures such as bars or breaks are not evident in the image, the number of components, that is, whether the galaxy is fitted with a single Sérsic (elliptical) or a bulge+disc (S0/0a), is decided through an automatic criteria. More details about this procedure can be found in Sect. 4.2.

Increasing the level of complexity of the galaxy model, by including extra components such as lenses, ovals, spiral arms, or barlenses, might improve the quality of the final fit, and provide extra information about the galaxy structure. However, it comes at the cost of greatly increasing the degeneracy on the final parameters, making their interpretation difficult. Therefore, we decided not to include any further structures in our analysis.

\subsection{Pre-processing SDSS images}

In order to perform the 2D photometric decomposition, GASP2D needs a series of input files: (1) a sky-subtracted image of the galaxy; (2) a mask created to avoid foreground stars, background galaxies, and other galactic features departing from the smooth light distribution of the galaxy; and (3) the radial profiles of ellipticity, position angle, and surface brightness. To compute such inputs, we make use of the fully-calibrated $g-, r$, and $i$-band images from the SDSS-DR7. 

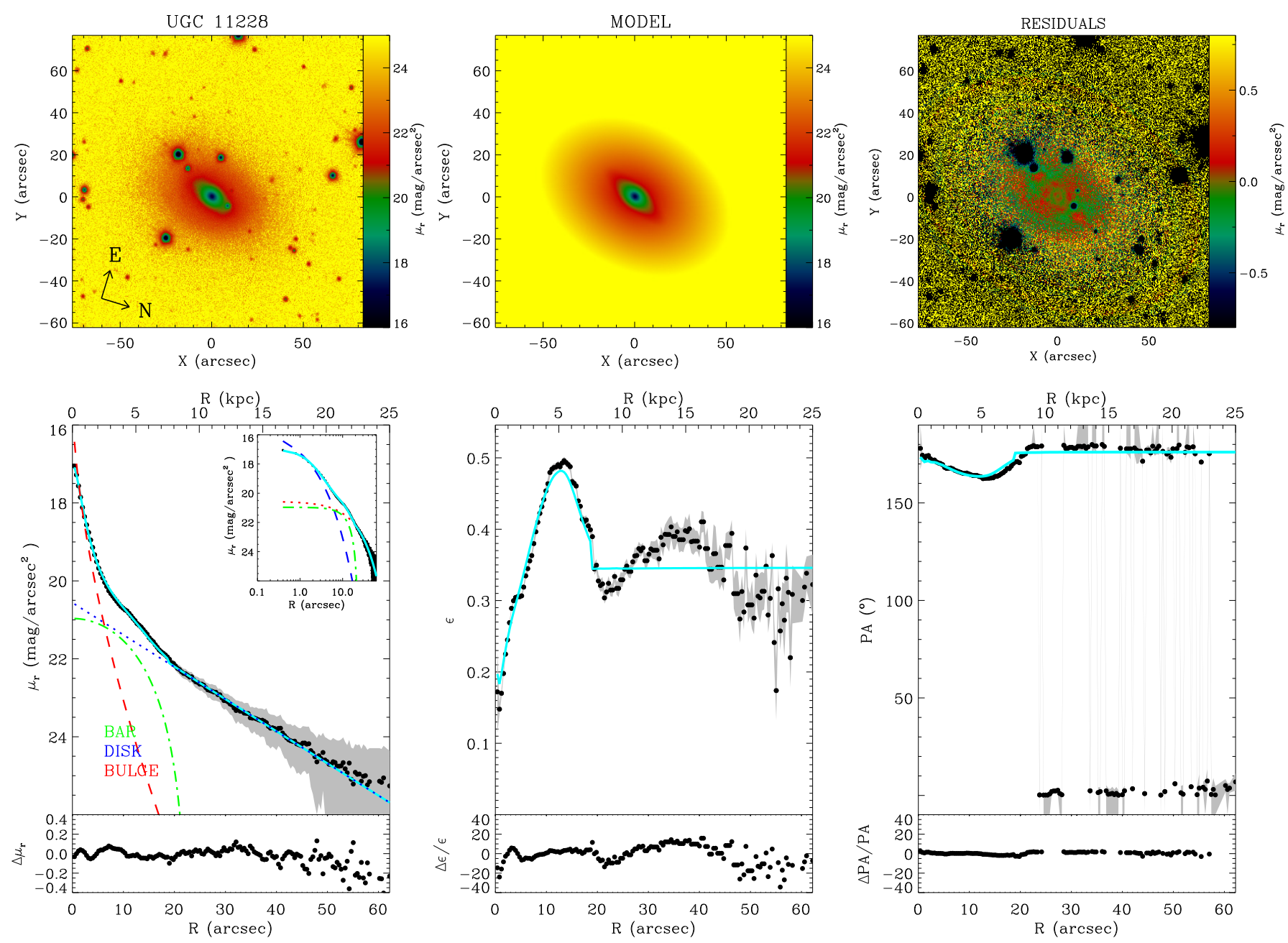

Fig. 3. Example of a diagnostic figure used to determine the number of stellar components for each galaxy. The plot represents the best fit using three components (bulge, bar, and disc) for the $r$-band image of UGC 11228. Similar plots were created for the $g$ - and $i$-band to check for consistency. Top left panel: galaxy image. Top middle panel: best-fitting model of the galaxy image obtained by adding a bulge, a bar, and a disc component. Top right panel: residual image obtained by subtracting the best-fit model from the galaxy image. Bottom left panel: ellipse-averaged surface brightness radial profile of the galaxy (black dots) and best-fit model (cyan solid line). The light contributions of the bulge (dashed red line), disc (dotted blue line), and bar (dotted-dashed green line) are shown. The upper inset shows a zoom of the surface-brightness data and fit with a logarithmic scale for the distance to the center of the galaxy. 1D surface brightness residuals (in mag/arcsec ${ }^{2}$ units) are shown in the bottom sub-panel. Bottom middle panel: ellipse-averaged radial profile of ellipticity of the galaxy (black dots) and best-fit model (cyan solid line). 1D residuals (in percentage) are shown in the bottom sub-panel. Bottom right panel: ellipse-averaged radial profile of position angle of the galaxy (black dots) and best-fit model (cyan solid line). 1D residuals (in percentage) are shown in the bottom sub-panel. The grey shaded areas in the bottom panels represent the measurement errors derived from the ellipse IRAF task when applied to the galaxy image.

\section{SDSS image sky subtraction, masks, and isophotal analysis}

The SDSS-DR7 used in this paper, as well as later SDSS data releases, provides a measurement of the sky level (usually the median value of every pixel in a frame of $\sim 13.51 \times 9.83 \mathrm{arcmin}^{2}$ after a sigma-clipping is applied). This estimate has been proven inadequate for some studies (Hyde \& Bernardi 2009), especially those focused on the analysis of the faintest parts of disc galaxies (Pohlen \& Trujillo 2006). Since we intend to provide a detailed inventory and characterisation of galaxy structures in the CALIFA survey, an improved sky subtraction procedure was needed. The details of this procedure are explained in a companion paper (Méndez-Abreu et al. 2016). For the sake of clarity we will briefly describe here its main characteristics. First, we created an ad hoc mask for each galaxy frame using both the automatic code SExtractor (Bertin \& Arnouts 1996) and a manually-built mask to include small features that SExtractor might have missed. Then, we use the ellipse IRAF $^{1}$ task to obtain the 1D surface-brightness profile using a fixed ellipticity and position angle matching the outermost disc isophotes. The actual value of the sky is then computed by averaging the region with a flat surface brightness at a radius where neither the galaxy nor other sources are affecting the flux. Finally, this value is subtracted from the corresponding science frame to produce the sky-subtracted image used in this paper. The distribution of the derived values of the surface brightness of the sky for the images of the sample galaxies are shown in the top panel of Fig. 4. We find typical values of $21.8 \pm 1.2,21.1 \pm 1.2$, and $20.2 \pm 1.2 \mathrm{mag} / \operatorname{arcsec}^{2}$ for $g-, r$-, and $i$-band, respectively.

1 IRAF is distributed by the National Optical Astronomy Observatory, which is operated by the Association of Universities for Research in Astronomy (AURA) under cooperative agreement with the National Science Foundation. 

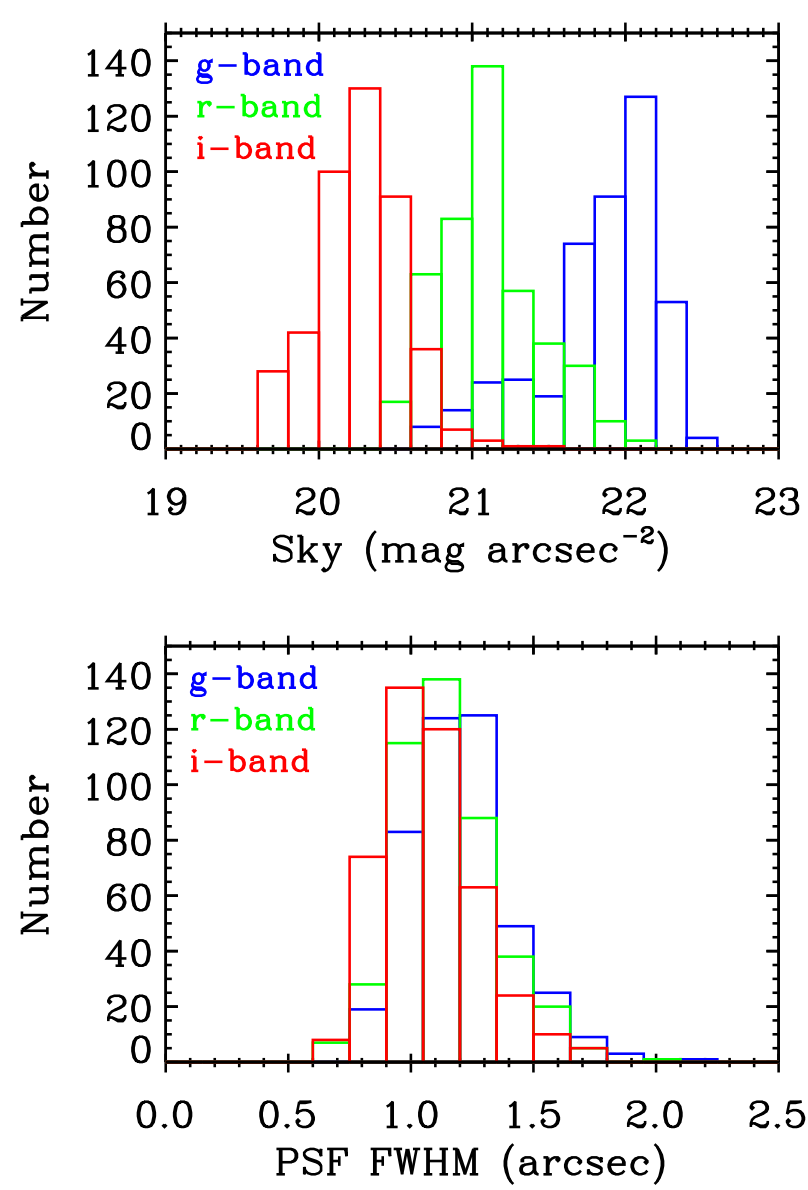

Fig. 4. Top panel: distribution of the average surface brightness of the sky subtracted from our galaxy images. Bottom panel: distribution of the PSF FWHM in our galaxy images. In both panels the blue, green, and red histograms represent the $g^{-}, r-$, and $i$-bands, respectively.

The final isophotal profiles used in this study were derived using the sky-subtracted images. We ran ellipse again allowing the isophotes to change the values of $\epsilon$ and PA to follow the galaxy morphology. The masks created during the sky subtraction process were used to derive these 1D profiles and in the actual 2D fit using GASP2D. Examples of the surface brightness, $\epsilon$, and PA 1D azimuthally averaged profiles are shown in Fig. 3.

\subsection{PSF}

It is well-known that accurate measurements of the image PSF are critical for recovery of the structural parameters of the galaxy central components. In this study this mainly refers to the properties of bulges and NPSs. Méndez-Abreu et al. (2008) found that errors of $\sim 2 \%$ in the PSF FWHM can produce variations of up to $10 \%$ in the $r_{\mathrm{e}}$ and $n$ bulge parameters. Similarly, Gadotti (2008) showed that the structural properties of bulges can be reliably retrieved provided that $r_{\mathrm{e}}$ is larger than $\sim 80 \%$ of the PSF half width half maximum (see also Costantin et al., in prep). Therefore, a careful analysis of the SDSS PSF is needed to perform a robust photometric decomposition.

We model the SDSS PSF with a Moffat function (see Eq. (3)). This parameterisation of the PSF has been extensively studied in the literature (e.g. Trujillo et al. 2001a) and provides an accurate representation of the SDSS PSF. For each galaxy image, a set of at least five non-saturated stars were fitted with a Moffat function using the IRAF task imexam. The mean values of the FWHM for our galaxy sample are shown in the bottom panel of Fig. 4. We find typical values of $1.2 \pm 0.2,1.1 \pm 0.2$, and $1.1 \pm 0.2$ arcsecs for the $g$-, $r$-, and $i$-band, respectively. GASP2D uses the measured PSF as kernel to be convolved with the model galaxy image before computing the $\chi^{2}$. This process is repeated in each iteration of the Levenberg-Marquardt minimisation process so that the final best-fit parameters are seeing-corrected.

\section{2D photometric decomposition analysis: multi-component fitting}

This section describes the main procedures we follow to carry out the photometric decomposition. We separate our sample into early-type (127 galaxies) and spiral galaxies (277 galaxies) since for the former an automatic methodology is used to find the optimal number of components to obtain the best fit whereas for the latter a human supervised approach is used. Table 1 shows the best fit parameters obtained for some example galaxies covering different combinations of structural components. The full version of the table for the entire galaxy sample is available on-line.

\subsection{Multi-wavelength fitting process}

The photometric properties of the different stellar structures were derived independently for the three SDSS bands $(g, r$, and $i$ ) used in this paper. This means that the structural parameters are not limited and/or tied between the different band images. However, in order to avoid discordant results we decided to relate the initial conditions required for the fit in the three bands. In its standard configuration, GASP2D finds the best set of initial conditions to initialise the non-linear fit in an automatic way. This procedure is described in Méndez-Abreu et al. (2008) and is mainly based on the analysis of the 1D radial profiles explained in Sect. 3.2. In this study, the galaxy images in the $r$-band (the intermediate wavelength band) were fitted following this standard procedure with automatic initial conditions or, in some cases, fine-tuning them after a visual inspection. Once a successful fit is achieved, the best-fit parameters in the $r$-band were used as initial conditions for the $g$ - and $i$-bands. We find that this strategy generally produces consistent results among different bands without constraining the final photometric parameters.

\subsection{One-component vs. multi-component fits of early-type galaxies}

The photometric properties of early-type galaxies, encompassing elliptical and lenticular galaxies, have been extensively studied in the literature (see Kormendy et al. 2009; Aguerri 2012, for reviews). However, the problem of identifying whether a stellar disc is present or not in these smooth and featureless galaxies is still under discussion (Gomes et al. 2016). From a photometric point of view, elliptical galaxies are stellar systems well described by a single Sérsic profile. On the other hand, the simplest description of a S0 galaxy is a two-component model, that is, a Sérsic profile describing the SBD of the bulge and a pure exponential representing the outer disc. It is worth noting that this definition is purely photometric and, therefore, is not directly related to the dynamical status of the galaxies (see Emsellem et al. 2011).

From a photometric point of view, advances in the field have been driven by the application of statistical techniques on model selection, such as the F-test (see Simard et al. 2011, for a similar application), the Bayesian inference criterion (BIC; Schwarz 1978), or the Akaike information criterion (AIC; Akaike 1974). 
Table 1. Structural parameters of the sample galaxies in the $r$-band.

\begin{tabular}{|c|c|c|c|c|c|c|}
\hline Galaxy & NGC 0155 & NGC 0001 & NGC 0160 & UGC 00036 & NGC 7819 & NGC 0941 \\
\hline$\mu_{\mathrm{e}}$ & $22.6 \pm 0.1$ & $19.8 \pm 0.2$ & $20.0 \pm 0.1$ & $19.1 \pm 0.3$ & $19.7 \pm 0.2$ & - \\
\hline$r_{\mathrm{e}}$ & $28.6 \pm 1.7$ & $4.6 \pm 0.5$ & $6.2 \pm 0.5$ & $1.4 \pm 0.3$ & $2.4 \pm 0.3$ & - \\
\hline$n$ & $5.0 \pm 0.2$ & $2.8 \pm 0.2$ & $2.6 \pm 0.1$ & $1.9 \pm 0.3$ & $1.1 \pm 0.1$ & - \\
\hline$q_{\text {bulge }}$ & $0.76 \pm 0.01$ & $0.80 \pm 0.02$ & $0.71 \pm 0.02$ & $0.71 \pm 0.05$ & $0.65 \pm 0.04$ & - \\
\hline $\mathrm{PA}_{\text {bulge }}$ & $174.1 \pm 0.6$ & $128.2 \pm 3.1$ & $49.5 \pm 4.2$ & $24.5 \pm 5.6$ & $88.7 \pm 5.2$ & - \\
\hline $\mathrm{B} / \mathrm{T}$ & 1.0 & 0.46 & 0.41 & 0.11 & 0.12 & - \\
\hline$\mu_{0}$ & - & $20.7 \pm 0.1$ & $21.55 \pm 0.03$ & $19.5 \pm 0.1$ & $22.1 \pm 0.1$ & $20.68 \pm 0.01$ \\
\hline$h$ & - & $14.9 \pm 0.9$ & $47.4 \pm 0.9$ & $9.5 \pm 0.4$ & $44.7 \pm 2.5$ & $20.2 \pm 0.4$ \\
\hline$r_{\text {break }}$ & - & - & $48.6 \pm 1.0$ & - & $46.9 \pm 4.4$ & - \\
\hline$h_{\text {out }}$ & - & - & $14.1 \pm 0.7$ & - & $12.1 \pm 1.5$ & - \\
\hline$q_{\text {disc }}$ & - & $0.62 \pm 0.01$ & $0.505 \pm 0.9$ & $0.45 \pm 0.01$ & $0.53 \pm 0.01$ & $0.84 \pm 0.01$ \\
\hline $\mathrm{PA}_{\text {disc }}$ & - & $96.5 \pm 1.1$ & $47.5 \pm 0.2$ & $17.9 \pm 0.7$ & $102.0 \pm 0.5$ & $166.0 \pm 0.7$ \\
\hline $\mathrm{D} / \mathrm{T}$ & - & 0.54 & 0.59 & 0.79 & 0.75 & 0.996 \\
\hline$\mu_{0, \text { bar }}$ & - & - & - & $20.5 \pm 0.1$ & $22.0 \pm 0.1$ & - \\
\hline$a_{\mathrm{bar}}$ & - & - & - & $12.4 \pm 0.3$ & $39.1 \pm 1.2$ & - \\
\hline$q_{\mathrm{bar}}$ & - & - & - & $0.63 \pm 0.01$ & $0.32 \pm 0.01$ & - \\
\hline $\mathrm{PA}_{\mathrm{bar}}$ & - & - & - & $134.0 \pm 0.4$ & $59.4 \pm 0.4$ & - \\
\hline $\operatorname{Bar} / T$ & - & - & - & 0.10 & 0.13 & - \\
\hline$\mu_{0, \mathrm{NPS}}$ & - & - & - & - & - & $18.2 \pm 0.1$ \\
\hline $\mathrm{NPS} / T$ & - & - & - & - & - & 0.004 \\
\hline Flag & $1, \mathrm{a}$ & $1, \mathrm{a}$ & $1, \mathrm{a}$ & $2, a$ & $2, \mathrm{c}$ & $2, \mathrm{c}$ \\
\hline
\end{tabular}

Notes. Best-fit parameters for a subsample of six galaxies modelled with a different combination of structures. From left to right: single Sérsic, bulge+disc, bulge+disc (including break), bulge+disc+bar, bulge+disc (including break)+bar, and NPS+disc. Each column represents the best-fit parameters for a given galaxy. From top to bottom: bulge parameters (effective surface brightness $\mu_{\mathrm{e}}$, effective radius $r_{\mathrm{e}}$, Sérsic index $n$, axis ratio $q_{\text {bulge }}$, position angle $\mathrm{PA}_{\text {bulge }}$, and bulge-to-total luminosity ratio $\mathrm{B} / \mathrm{T}$ ), disc parameters (central surface brightness $\mu_{0}$, inner scale length $h$, break radius $r_{\text {break }}$, outer scale length $h_{\text {out }}$, axis ratio $q_{\text {disc }}$, position angle $\mathrm{PA}_{\text {disc }}$, and disc-to-total luminosity ratio $\mathrm{D} / \mathrm{T}$ ), bar parameters (central surface brightness $\mu_{0, \text { bar }}$, bar radius $a_{\text {bar }}$, axis ratio $q_{\mathrm{bar}}$, position angle $\mathrm{PA}_{\mathrm{bar}}$, and bar-to-total luminosity ratio Bar/T), NPS parameters (central surface brightness $\mu_{0, \mathrm{NPS}}$ and NPS-to-total luminosity ratio NPS/T), and the visual quality flag explained in Sect. 5.1. Each row shows the best fitting values of the given parameters and their associated error (see Sect. 5.2). Surface brightness, radii, and position angles are given in units of $\mathrm{mag} / \operatorname{arcsec}^{2}$, arcsec, and degrees measured counterclockwise from North to East, respectively. When a given structure is not present in the model, its corresponding parameters are left empty. The full version of the table is available on-line. Similar tables are also available for the $g$ - and $i$-bands. The parameters in this table have not been corrected for galactic extinction, K-correction, or cosmological dimming.

These techniques work by adding a penalisation to the standard $\chi^{2}$ accounting for the number of free parameters included in the fit. Thus, these criteria can be applied to determine whether or not adding an extra component (i.e. an outer disc) would statistically improve the best fit (e.g. Simard et al. 2011; Head et al. 2014). On the other hand, these automatic criteria do not account for the possibility that, even if a given model is statistically preferred, its solution might be unphysical. Therefore, to provide the best mathematical fit with a physical meaning, some authors have proposed the use of a "logical filter" (Allen et al. 2006). In Méndez-Abreu et al. (2016) we combined the two aforementioned approaches to assess the appropriateness of different model decompositions and decide when a complex model, bulge+disc, is preferred over a single Sérsic profile. The main features of the logical filter consist of classifying as ellipticals those galaxies where two-component models produce either: i) a large $\mathrm{B} / \mathrm{T}(\mathrm{B} / \mathrm{T}>0.9)$; ii) a large $r_{\mathrm{e}}\left(r_{\mathrm{e}}>1.676 \times h\right)$; iii $)$ an even number ( 0 or 2 ) of crossing points between the surfacebrightness distribution of the disc and the bulge; or iv) the previous crossing point happening before one effective radius of the disc $\left(r_{\text {cross }}<1.676 \times h\right)$. Note that galaxies hosting stellar bars are relatively easy to identify. Since bars can be used to detect the presence of stellar discs (Méndez-Abreu et al. 2010, 2012),
Table 2. Schematic of the sample selection process of early-type galaxies.

\begin{tabular}{cccc}
\hline \hline $\begin{array}{c}\text { CALIFA visual } \\
(1)\end{array}$ & $\begin{array}{c}\text { L. Filter } \\
(2)\end{array}$ & $\begin{array}{c}\text { L. Filter }+\Delta \mathrm{BIC} \\
(3)\end{array}$ & $\begin{array}{c}\text { Final } \\
(4)\end{array}$ \\
\cline { 1 - 3 } $85 \mathrm{E}$ & $35 \mathrm{E}$ & $35 \mathrm{E}$ & \\
& $50 \mathrm{~S} 0$ & $\begin{array}{c}30 \mathrm{~B} / \mathrm{BD} \\
20 \mathrm{~S} 0\end{array}$ & $41 \mathrm{E}$ \\
& $6 \mathrm{E}$ & $6 \mathrm{E}$ & $36 \mathrm{BD}$ \\
$42 \mathrm{~S} 0$ & $36 \mathrm{~S} 0$ & $6 \mathrm{~B} / \mathrm{BD}$ & \\
\cline { 2 - 3 } & & $30 \mathrm{~S} 0$ & \\
\hline
\end{tabular}

Notes. E-Elliptical, S0-Lenticular, B/BD-Elliptical or Lenticular. (1) Number of galaxies using the CALIFA visual classification; (2) number of galaxies after the logical filtering (L. Filter); (3) number of galaxies after the logical filtering and BIC classification; (4) final classification used in this study.

they are directly classified as S0 without the need to satisfy either the logical filtering or BIC criteria.

We therefore applied the two-step process described in Méndez-Abreu et al. (2016) to our sample of 127 early type galaxies. Table 2 shows the outcome of our analysis. Galaxies were first divided into ellipticals and $\mathrm{S} 0$ based on the outcome of 
the logical filter. Then, for those galaxies where the two models are compatible, the BIC analysis is performed to discern whether there is statistical evidence against one of the models or not. Still, some galaxies remain with unclear classification, equally compatible with being a single Sérsic or a two-component galaxy. This last sample is labelled in the tables as B/BD morphology and highlights the intrinsic difficulties of separating ellipticals from S0 galaxies using photometric data. The best-fit obtained from both the single Sérsic and the bulge+disc is provided for these galaxies to allow the users to decide which decomposition is more suitable for their science case. We end up with a final sample of 41 ellipticals, 50 lenticulars, and 36 galaxies with B/BD morphology.

\subsection{Multi-component analysis of spiral galaxies}

The final decomposition of our CALIFA sample of spiral galaxies was done using a human-supervised approach fitting up to three components: bulge/NPS, disc (including break), and bar. For each galaxy, a given combination of these structures provides the best fit.

Small bulges with sizes comparable to the SDSS PSF can lead to erroneous fits, usually producing extreme values of the Sérsic index $(n>7)$. Although one of our goals is to produce reliable estimates of the bulge parameters, high values of $n$ might also have a significant impact on other components' parameters. Therefore, we also fit these galaxies using a NPS instead of the Sérsic parameterisation for the bulge. A visual inspection of the $2 \mathrm{D}$ residual is then used to determine which component is preferred. As a consequence, a bulge and a NPS would never be fitted simultaneously in the same galaxy, and galaxies better represented by a NPS should not be understood as necessarily hosting a nuclear star cluster or AGN. GASP2D also allows for different behaviours of the outer disc component (exponential profile, Type I; and broken profiles, Type II and Type III). Our CALIFA sample was fitted using 13 different combinations of the previous structures (see Table 3). This demonstrates the morphological variety of our galaxies and the necessity of performing accurate multi-component photometric decompositions.

In Sect. 4.2 we described the particular case of fitting earlytype galaxies. The detection of stellar discs in spiral galaxies is more direct than for early-type galaxies due to the presence of star formation following the characteristic spiral pattern. The strategy followed to perform the photometric decomposition of a spiral galaxy starts with a two-component bulge+disc fit. Right after, or from the start if its presence is readily apparent in the galaxy image, we check for the addition of a bar component (see Sect. 4.3.1). If the bar is not obvious in the galaxy image, both the bulge+disc and bulge+disc+bar fittings are performed. Then, a careful visual analysis of the 2D SBD residual, as well of the 1D ellipticity and PA profiles (see Aguerri et al. 2009, for a description of bar identification through the galaxy ellipticity and PA) is carried out to reveal whether a bar is actually present or not. It is worth noting that the detection of bars is limited to those central structures with an axis ratio $q_{\mathrm{bar}}<0.7$. The next step is to check for the presence of broken discs and, if the following conditions are fulfilled, include them in the fit: i) after a careful revision of the 2D SBD residual we confirm potential breaks are not misidentified with spiral arms and/or outer rings; and ii) they appear at a SB level $\mu_{i}<24 \mathrm{mag} / \operatorname{arcsec}^{2}$ so they can be robustly measured with the SDSS imaging (see Sect. 5.2).

The different combinations of structures used in our final galaxy sample are shown in Table 3 . In the following sections
Table 3. Different combinations of structures present in the photometric decomposition of our sample galaxies.

\begin{tabular}{|c|c|}
\hline $\begin{array}{l}\text { Structure } \\
\text { (1) }\end{array}$ & $\begin{array}{c}\text { Number } \\
\text { (2) }\end{array}$ \\
\hline $\mathrm{B}$ & 43 \\
\hline $\mathrm{D}$ & 6 \\
\hline $\mathrm{BD}$ & 74 \\
\hline ND & 12 \\
\hline $\mathrm{BD}_{\mathrm{br}}$ & 67 \\
\hline $\mathrm{D}_{\mathrm{br}}$ & 4 \\
\hline BDBar & 88 \\
\hline DBar & 7 \\
\hline NDBar & 10 \\
\hline $\mathrm{BD}_{\mathrm{br}} \mathrm{Bar}$ & 47 \\
\hline $\mathrm{D}_{\mathrm{br}} \mathrm{Bar}$ & 3 \\
\hline $\mathrm{ND}_{\mathrm{br}} \mathrm{Bar}$ & 5 \\
\hline BDBarNBar & 2 \\
\hline $\mathrm{B} / \mathrm{BD}$ & 36 \\
\hline
\end{tabular}

Notes. (1) Type of structure: B - single Sérsic, D - pure disc, BD - bulge+disc, ND - nuclear point source+disc, $\mathrm{BD}_{\mathrm{br}}$ - bulge+disc with break, $\mathrm{D}_{\mathrm{br}}$ - pure disc with break, BDBar - bulge+disc+bar, DBar - disc+bar, NDBar - nuclear point source+disc+bar, BD $\mathrm{br}_{\mathrm{bar}}$ - bulge+disc with break+bar, $\mathrm{D}_{\mathrm{br}} \mathrm{Bar}$ - disc with break+bar, $\mathrm{ND}_{\mathrm{br}} \mathrm{Bar}$ - nuclear point source+disc with break+bar, BDBarNBar bulge+disc+bar+nuclear bar, B/BD - either single Sérsic or bulge+disc; (2) number of galaxies.

we will describe the incidence of the different galaxy structures in the CALIFA sample.

\subsubsection{Barred galaxies}

The inclusion of the bar SBD in the photometric decomposition has been proven to be critical in order to recover accurate bulge parameters (e.g. Aguerri et al. 2005; Laurikainen et al. 2005). Several studies have shown that both the Sérsic index and the $\mathrm{B} / \mathrm{T}$ ratio can artificially increase if the bar is not properly accounted for in the fit (Gadotti 2008; Salo et al. 2015).

It is worth noting that the inclusion of a stellar bar in our photometric decomposition is independent of the visual morphological classification (see Walcher et al. 2014, for details). Therefore, we can quantify the impact of different bar classification methods on studies of the galaxy bar fraction. Figure 5 shows the distribution of the bar fraction using either the visual classification or the photometric decomposition method with respect to the galaxy Hubble type and stellar mass. In addition, we have included the volume corrected bar fractions for the photometric decomposition method applied to those galaxies extracted from the CALIFA mother sample (see Sect. 2). A summary of the result is presented in Table 4. In general, we find a good agreement in the observed bar fractions obtained using the visual and photometric decomposition method. The uncertainty in the visual identification of a bar is enough to account for the differences with respect to the photometric decompositions for all cases except for the galaxies classified as S0 and Sd. In the case of the S0, not only the presence of a bar but also the morphological classification as S0 itself depends on the method (see Sect. 4.2). We attribute the differences in the Sd galaxies to the small number statistics (only 24 galaxies are $\mathrm{Sd}$ ). The volume corrected fractions can deviate substantially from the estimates from the photometric decomposition method. 


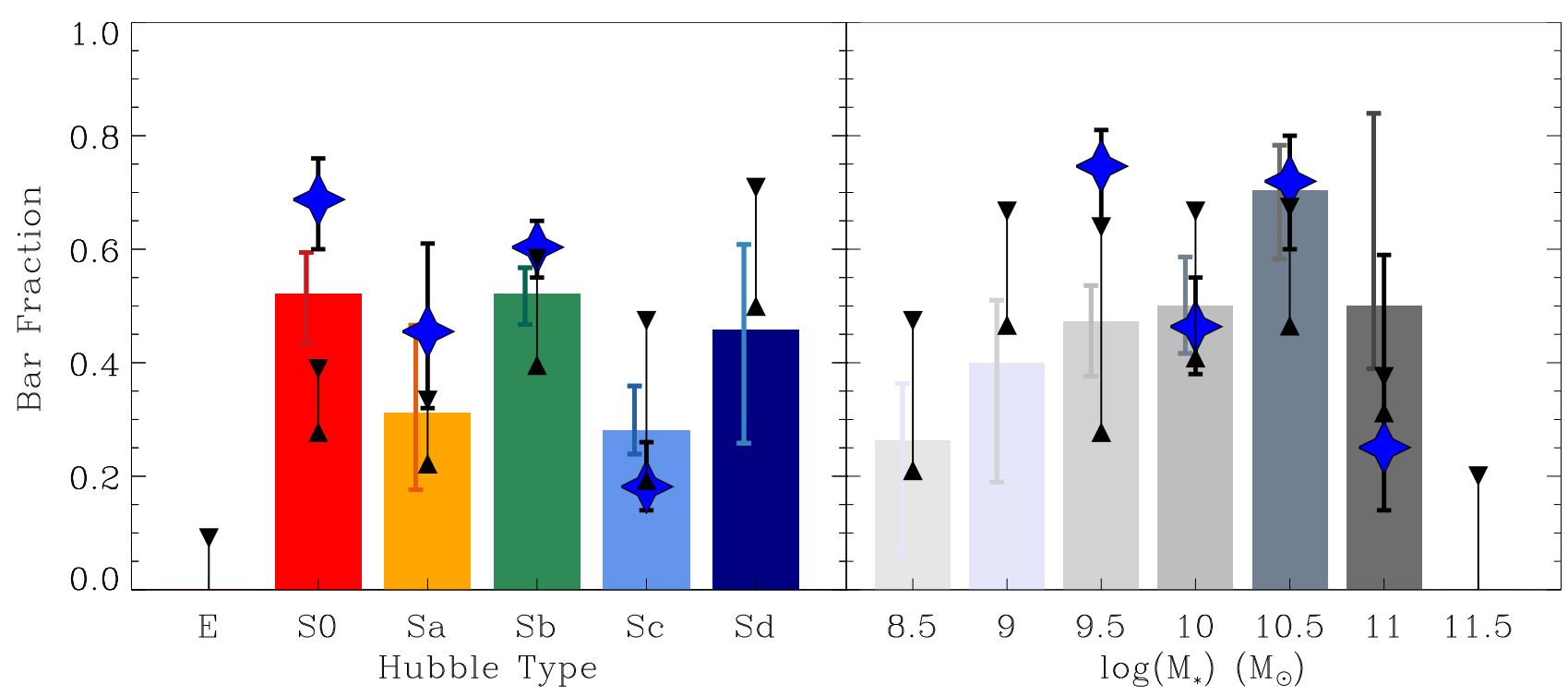

Fig. 5. Distribution of the bar fraction as a function of the Hubble type (left panel) and stellar mass (right panel). Colour bars represent the photometric bar fraction derived from this study. Lines with darker colours show the $1 \sigma$ error. Blue stars show the volume corrected bar fractions using only those galaxies drawn from the mother sample and with luminosities within the CALIFA completeness limits (285 galaxies). The black lines represent the visual classification from Walcher et al. (2014) with the upper limits including both weak (AB) and strong (B) bars and the lower limit accounting only for strong bars. Note that since the Walcher et al. (2014) Hubble type and bar identification are the average among different independent classifications, some ellipticals were classified as weakly barred. Bins with less than five galaxies are not shown.

Table 4. Volume corrected bar fraction distributions in our galaxy sample.

\begin{tabular}{lc}
\hline \hline Hubble type & Bar fraction \\
\hline S0 & $68.7 \% \pm 7.2 \%$ \\
Sa & $45.5 \% \pm 15.5 \%$ \\
Sb & $60.3 \% \pm 4.7 \%$ \\
Sc & $18.2 \% \pm 7.8 \%$ \\
Sd & - \\
\hline Stellar mass & Bar fraction \\
\hline $9<\log \left(M_{\star} / M_{\odot}\right)>9.5$ & - \\
$9.5<\log \left(M_{\star} / M_{\odot}\right)>10$ & $74.6 \% \pm 6.4 \%$ \\
$10<\log \left(M_{\star} / M_{\odot}\right)>10.5$ & $46.4 \% \pm 8.6 \%$ \\
$10.5<\log \left(M_{\star} / M_{\odot}\right)>11$ & $72.0 \% \pm 8.0 \%$ \\
$11<\log \left(M_{\star} / M_{\odot}\right)>11.5$ & $25.1 \% \pm 22.1 \%$ \\
\hline
\end{tabular}

The influence of galaxy morphology on the bar fraction has been extensively discussed in the literature with contradictory results. Several authors have claimed that the bar fraction increases towards early-type galaxies (Masters et al. 2011, 2012; Lee et al. 2012) with this trend being consistent with some models of bar formation and evolution (Athanassoula et al. 2013). Nevertheless, other studies have found the opposite trend with Hubble type (Laurikainen et al. 2007; Barazza et al. 2008; Aguerri et al. 2009; Buta et al. 2015). Our volume-corrected bar fraction is relatively constant for early-types (S0, Sa, and Sb) but it dramatically drops for Sc galaxies. Unfortunately, small number statistics for the Sd type preclude further analysis of that bin, so we cannot confirm the drop in the bar fraction for all late-type galaxies. We find a mean value for the volume-corrected bar fraction of $57 \%$. This number is similar to that obtained using the observed sample (54\%) and the visual classification (51\%). Recently, Buta et al. (2015) found a lower bar fraction in early-type galaxies $(\sim 56 \%)$ than in late-type galaxies $(80 \%)$ using a visual classification of the S4G galaxy sample (Sheth et al. 2010). They suggested that the different mass distribution of galaxies with both early- and late-type morphologies could, however, be driving this result.

In fact, the previous, apparently contradictory results can be reconciled when the galaxy stellar mass is accounted for in the sample selection (Nair \& Abraham 2010). The incidence of bars in galaxy discs is a strong function of stellar mass with a maximum bar fraction at $M_{\star} \sim 2 \times 10^{9} M_{\odot}$ for field galaxies (Méndez-Abreu et al. 2010, 2012). The volume corrected fractions show an increase of the bar fraction towards lower stellar masses. This trend is in good agreement with the results of Méndez-Abreu et al. (2012) although the CALIFA sample is not complete at $M_{\star} \sim 10^{9} M_{\odot}$ where the bar incidence is predicted to be highest. The observed bar fraction shows a different picture, being nearly independent of stellar mass within the errors for both the visual and photometric decomposition method.

An interesting sub-sample of barred systems contains nested bars, that is, galaxies hosting both a large-scale bar (described previously) and an inner, secondary bar embedded in the main one (Erwin \& Sparke 2002; Corsini et al. 2007; de Lorenzo-Cáceres et al. 2012, 2013). Whilst they are not the main focus of this paper, they are thought to be present in $\sim 30 \%$ of barred galaxies (Laine et al. 2002; Erwin 2004) and might have an impact on the bulge parameters (de Lorenzo-Cáceres et al., in prep.). Therefore, when necessary we included a secondary bar (using another Ferrers profile) in our photometric decomposition. We found only two galaxies with nested bars; NGC 0023 and NGC 7716, which represent a much lower fraction $(1.5 \%)$ with respect to previous findings. Angular resolution likely explains these differences. The sample presented in Erwin (2004) is located at an average distance of $29 \mathrm{Mpc}$ $(0.144 \mathrm{kpc} / \mathrm{arcsec})$, with some of the inner bars detected by using imaging from the Hubble Space Telescope Wide Field Planetary Camera 2 (HST/WFPC2) with a typical 0.07 arcsec resolution. On the other hand, our sample of barred galaxies is located at $72 \mathrm{Mpc}(0.346 \mathrm{kpc} / \mathrm{arcsec})$ limited to the $\sim 1.1 \mathrm{arcsec}$ SDSS resolution. Thus, in our sample a low double bar fraction is expected. 


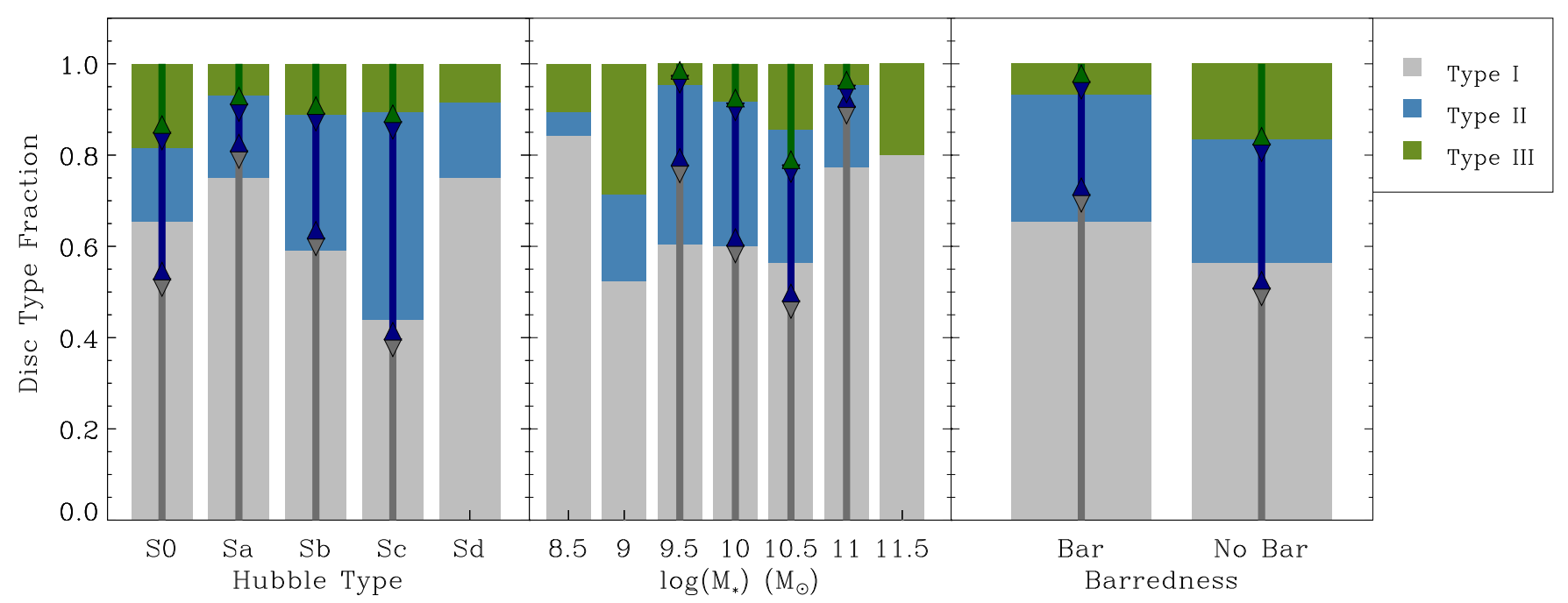

Fig. 6. Distribution of the three different disc profiles used in this study with Hubble type (left panel), stellar mass (middle panel), and presence of a bar (right panel). Colour bars represent our observed fractions for Type I (grey), Type II (blue), and Type III (green). Lines with darker colours show the volume-corrected fractions. Bins with less than five galaxies are not represented.

\subsubsection{Breaks in disc galaxies}

Galactic discs with non-purely exponential profiles represent an important fraction of the discs in the Local Universe and are known to appear in galaxies independently of their Hubble type (e.g. Erwin et al. 2008; Gutiérrez et al. 2011; Marino et al. 2016). The ubiquity of stellar discs with broken profiles is manifest even at high redshift (Pérez 2004; Trujillo \& Pohlen 2005; Azzollini et al. 2008), suggesting that they are key features in understanding how galaxies form and evolve.

From the photometric decomposition point of view, broken profiles have an impact on the properties of the remaining components included in the fit. Therefore, a complete and robust analysis of the photometric properties of galaxies must include the possibility of disc galaxies displaying broken profiles. Previous studies of the light distribution in the galaxy outskirts have mainly been based on the analysis of 1D azimuthally averaged profiles, and thus are not representative of the two-dimensional nature of the problem of galaxies. GASP2D is able to perform a multi-component decomposition of the galactic light including broken profiles and this work is a pioneering study on the incidence of broken profiles in disc galaxies based on the 2D approach.

Figure 6 shows the distribution of the different disc profiles as a function of the Hubble type, stellar mass, and presence of a bar. We find that $62 \%, 28 \%$, and $10 \%$ of our volume corrected disc sample is best represented with a Type I, Type II, and Type III, respectively. This represents a significantly lower fraction of broken discs when compared to 1D analyses present in either the literature (e.g. Gutiérrez et al. 2011; Laine et al. 2014) or the CALIFA sample (Marino et al. 2016). In Ruiz-Lara et al. (in prep.) we performed a detailed analysis to understand these differences and concluded that the discrepancy is mainly caused by our new 2D approach instead of due to selection effects in the galaxy samples. In general, multi-component 2D decompositions such as the one performed in this paper build the galaxy model as a combination of different galaxy structures that contribute differently to the total luminosity depending on the galactocentric radius. This is important in regions with a high overlap of structures such as the area where the bulge, disc, and bar coexist. On the contrary, most of the previous studies using
1D profiles used pre-defined regions of the surface-brightness profile where a single exponential is fitted, without taking into account any superposition with other galaxy components (see Muñoz-Mateos et al. 2013, for an estimation of errors associated to the pre-defined disc regions). This different methodology leads to a higher fraction of broken profiles in 1D analysis (in particular Type II), since it is straightforward to accommodate piecewise exponentials to different sections of the profile, but it is not straightforward to associate these piecewise exponentials with an actual change in disc structure. At the same time, Type III breaks usually occur at lower surface brightness than Type II (Pohlen \& Trujillo 2006) so they are intrinsically more difficult to identify. In 2D multi-component analysis the addition of this new structure (i.e. two new free parameters $r_{\text {break }}$ and $h_{\text {out }}$ ) is not always justified in statistical terms (see Sect. 5.2), whereas $1 \mathrm{D}$ analysis methods can easily deal with fitting a pre-defined region even if at low $\mathrm{SB}$.

The differences of this work with respect to previous studies lie not only in the different techniques (1D vs. 2D), but also in the different samples under analysis. The sample analysed in Pohlen \& Trujillo (2006) consists of 94 late-type spiral galaxies (11\% Type I, $66 \%$ Type II, and 33\% Type III) whereas Erwin et al. (2008) and Gutiérrez et al. (2011) analysed 66 barred (27\% of Type I, $42 \%$ Type II, and 24\% Type III) and 47 unbarred (28\% of Type I, $21 \%$ Type II, and $51 \%$ Type III) early-type galaxies, respectively. Figure 6 shows that, according to our 2D approach, the fraction of Type I profiles decreases with later Hubble types whereas the fraction of Type II profiles increases (with the exception of Sd galaxies) in agreement with previous findings using 1D analyses (although with different fractions). For Type III profiles we find that the fraction of galaxies displaying this profile remains constant with Hubble type. No significant trends are found in terms of the stellar mass or the presence of a bar in agreement with Marino et al. (2016). A summary of our results is shown in Table 5.

\subsubsection{NPS and pure disc galaxies}

There is compelling evidence that low $\mathrm{B} / \mathrm{T}$ and pure disc galaxies are common in the Local Universe, especially in low-mass or late-type galaxies (Böker et al. 2002; Salo et al. 2015). Recent 
Table 5. Volume corrected disc type distributions in our galaxy sample.

\begin{tabular}{lccc}
\hline \hline Hubble type & Type I & Type II & Type III \\
\hline S0 & $52.8 \%$ & $32.1 \%$ & $15.1 \%$ \\
Sa & $80.8 \%$ & $10.3 \%$ & $8.9 \%$ \\
Sb & $61.7 \%$ & $27.3 \%$ & $11.0 \%$ \\
Sc & $39.6 \%$ & $47.7 \%$ & $12.7 \%$ \\
Sd & - & - & - \\
\hline Stellar mass & Type I & Type II & Type III \\
\hline $9<\log \left(M_{\star} / M_{\odot}\right)>9.5$ & - & - & - \\
$9.5<\log \left(M_{\star} / M_{\odot}\right)>10$ & $77.7 \%$ & $21.7 \%$ & $0.6 \%$ \\
$10<\log \left(M_{\star} / M_{\odot}\right)>10.5$ & $60.1 \%$ & $33.1 \%$ & $6.7 \%$ \\
$10.5<\log \left(M_{\star} / M_{\odot}\right)>11$ & $48.0 \%$ & $31.8 \%$ & $20.2 \%$ \\
$11<\log \left(M_{\star} / M_{\odot}\right)>11.5$ & $90.6 \%$ & $6.6 \%$ & $2.8 \%$ \\
\hline Barredness & Bar fraction \\
\hline Bar & $71.2 \%$ \\
No Bar & $50.8 \%$ & $24.8 \%$ & $3.9 \%$ \\
\hline
\end{tabular}

Table 6. Volume corrected B/T distributions in our galaxy sample.

\begin{tabular}{lc}
\hline \hline Hubble type & $\langle\mathrm{B} / \mathrm{T}\rangle$ \\
\hline S0 & $0.33 \pm 0.16$ \\
Sa & $0.24 \pm 0.17$ \\
Sb & $0.12 \pm 0.11$ \\
Sc & $0.05 \pm 0.09$ \\
Sd & $0.02 \pm 0.05$ \\
\hline Stellar mass & $\langle\mathrm{B} / \mathrm{T}\rangle$ \\
\hline $9<\log \left(M_{\star} / M_{\odot}\right)>9.5$ & $0.05 \pm 0.13$ \\
$9.5<\log \left(M_{\star} / M_{\odot}\right)>10$ & $0.05 \pm 0.09$ \\
$10<\log \left(M_{\star} / M_{\odot}\right)>10.5$ & $0.15 \pm 0.14$ \\
$10.5<\log \left(M_{\star} / M_{\odot}\right)>11$ & $0.22 \pm 0.13$ \\
$11<\log \left(M_{\star} / M_{\odot}\right)>11.5$ & $0.29 \pm 0.13$ \\
\hline
\end{tabular}

works using large galaxy samples drawn from the SDSS survey have found that $15 \%-20 \%$ of disc galaxies out to $z \sim 0.03$ appear as pure discs (Kautsch et al. 2006; Barazza et al. 2008; Kautsch 2009). The high observational incidence of both pure discs and low $\mathrm{B} / \mathrm{T}$ galaxies in the Local Universe is challenging for cosmological galaxy formation simulations to reproduce (Abadi et al. 2003; Governato et al. 2004; Peebles \& Nusser 2010) and only recently, galaxies with realistic $\mathrm{B} / \mathrm{T}$ distributions have been obtained (see Brooks \& Christensen 2016, for a review).

Figure 7 shows the distribution of $\mathrm{B} / \mathrm{T}$ as a function of the Hubble type and galaxy mass. The relative size of the bulge with respect to the galaxy is one of the primary features that distinguishes different Hubble types, therefore the drop of B/T from early- to late-type galaxies is not surprising. Our result is also quantitatively in agreement with previous works in the literature. Laurikainen et al. (2010) found a decline in the mean B/T values from 0.32 to 0.07 for $\mathrm{S} 0$ and $\mathrm{Sd}$ galaxies, respectively. Similar values were more recently obtained by Salo et al. (2015) and are consistent with our volume-corrected measurements of 0.33 and 0.02 for S0 and Sd galaxies, respectively (see Table 6 for a summary of the $\mathrm{B} / \mathrm{T}$ values). We also find a clear trend of the $\mathrm{B} / \mathrm{T}$ ratio increasing with the galaxy stellar mass for the volume-corrected sample (right panel in Fig. 7). Nonetheless, these two relations are not completely independent since, in our sample, later Hubble types are systematically less massive than early-types (see Fig. 8). This trend between the Hubble type and stellar mass holds for the observed, but also for the volume corrected quantities, and has already been found in the literature (Huertas-Company et al. 2011; Torres-Papaqui et al. 2012).
We find that $24 \%$ and $76 \%$ of our volume-corrected disc galaxies have $\mathrm{B} / \mathrm{T}>0.2$ and $\mathrm{B} / \mathrm{T}<0.2$, respectively. The relative fraction of galaxies with different $\mathrm{B} / \mathrm{T}$ imposes strong constraints on the galaxy evolution scenarios. Weinzirl et al. (2009) found that $34 \%$ and $66 \%$ of their high-mass $\left(\log \left(M_{\star} / M_{\odot}\right)>10\right)$ spiral sample had $\mathrm{B} / \mathrm{T}>0.2$ and $\mathrm{B} / \mathrm{T}<0.2$, respectively. Limiting our sample to this mass limit we find $36 \%$ and $64 \%$ of our volume-corrected disc galaxies have $\mathrm{B} / \mathrm{T}>0.2$ and $\mathrm{B} / \mathrm{T}<0.2$, respectively, in remarkably good agreement with their results.

Figure 9 shows the fraction of the final decomposition models used to represent our galaxy sample as a function of the stellar mass. Here we focus on the incidence of bulge components in the photometric decomposition, and therefore we group together all possible model combinations (see Table 1) in five groups depending only on their central component, and neglecting other structures such as broken discs or bars: i) galaxies well represented by a single Sérsic model (42 ellipticals); ii) bulge+disc galaxies with $\mathrm{B} / \mathrm{T}>0.2$ (91 galaxies); iii) bulge+disc galaxies with $\mathrm{B} / \mathrm{T}<0.2$ (184 galaxies); iv) galaxies with no bulge and pure disc (22 galaxies); and v) galaxies with a NPS+disc (27 galaxies). Figure 9 shows a clear segregation of galaxy types depending on their stellar mass. The fraction of single Sérsic (i.e. ellipticals) and bulge+disc with $\mathrm{B} / \mathrm{T}>0.2$ galaxies steadily increase with galaxy mass. Indeed they are the dominant population of galaxies with $M_{\star}>10^{11} M_{\odot}$. On the other hand, bulge+disc with $\mathrm{B} / \mathrm{T}<0.2$ galaxies are the preponderant galaxy population in the mass range $10^{9.5}<M_{\star} / M_{\odot}<10^{11}$, whilst galaxies with negligible bulges, both pure disc and NPS+disc models, prevail at low masses $M_{\star}<10^{9} M_{\odot}$.

\section{Quality assessment and uncertainties in the photometric decomposition}

Determining the uncertainties in the photometric parameters in multi-component decompositions such as the one presented in this paper is a complicated task. In this section, we describe our attempts to provide meaningful uncertainties for the different parameters using three different approaches: a visual quality control analysis, a quantitative error measurement based on mock galaxies, and a comparison with previous works in the literature.

\subsection{Visual quality check of the fits}

The galaxy sample analysed in this paper was drawn from the CALIFA DR3 sample by applying two main criteria: i) they are not interacting or merging and ii) they are relatively faceon $(i \lesssim 70)$. However, there could still be issues related to either the original SDSS imaging, the presence of strong extra components not included in the fit (spiral arms or rings), or difficulties inherent to the fitting process that can compromise the quality of the final decomposition. In order to assess the reliability of the fits, and to quantify the incidence of these issues in our galaxy sample, we carried out a visual check of each individual fit assigning a "quality flag" that is provided in the tables together with the results of the decompositions.

The quality flag is defined as a number $(1,2,3$, or 4$)$ followed by a letter $(a, b$, or $c)$. Different numbers correspond to galaxies with (1) both good imaging and no other strong extra galaxy components affecting the fit; (2) good imaging but strong extra components that might influence the final fit; (3) poor imaging but no strong extra components affecting the final fit; and (4) poor imaging and strong extra components affecting the final fit. We refer to problems with the original imaging as 


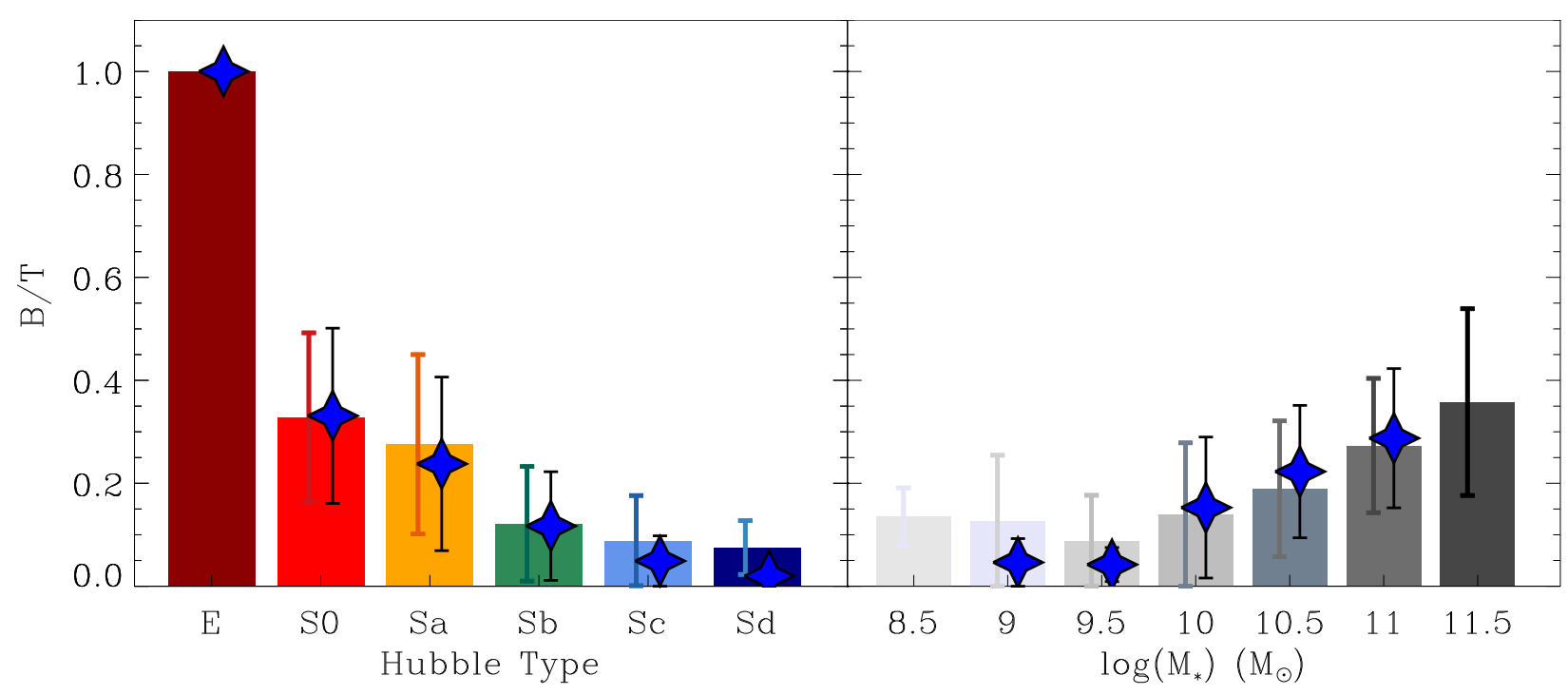

Fig. 7. Distribution of the $\mathrm{B} / \mathrm{T}$ ratio with Hubble type (left panel) and stellar mass (right panel). Colour bars represent the mean values of B/T for the observed distributions. Lines with darker colours show the $1 \sigma$ error. Blue stars show the volume weighted mean values of the $\mathrm{B} / \mathrm{T}$ ratios with its corresponding $1 \sigma$ errors. Elliptical galaxies have been excluded from the distribution in the right panel. Bins with less than five galaxies are not represented.

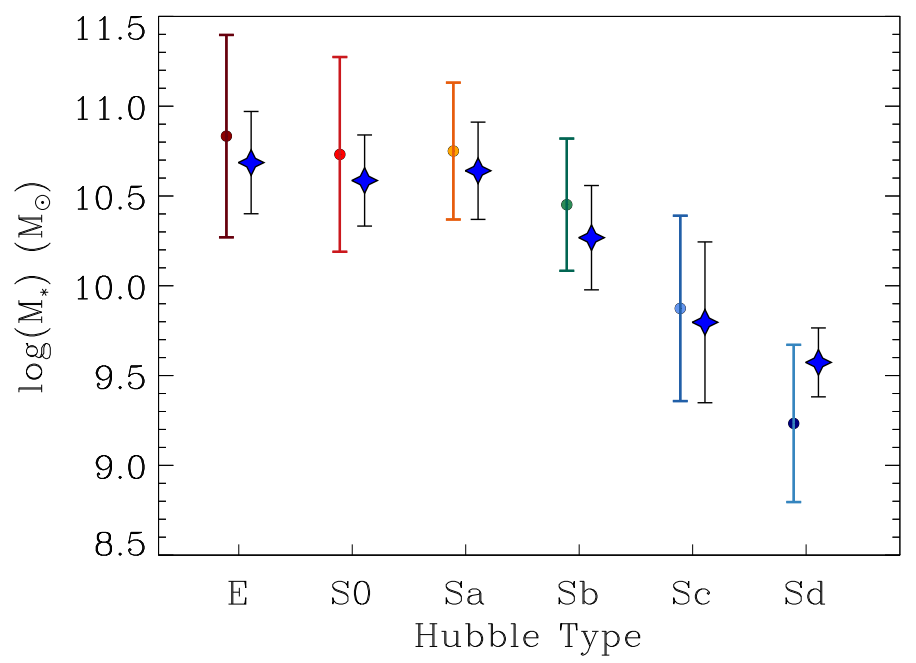

Fig. 8. Distribution of galaxy stellar mass with Hubble type for our galaxy sample. Colour points and bars represent the mean values and $1 \sigma$ error of stellar mass for the observed distributions. Blue stars show the volume-weighted mean values of the mass with its corresponding $1 \sigma$ errors.

those due to the presence of other bright galaxies whose stellar haloes overlap with our galaxy, and/or strong fluctuations of the local sky background around the galaxy. The different letters are related to the fitting process. Due to the highly degenerate parameter space we are dealing with (some fits include up to 17 free parameters), in some cases it was not possible to achieve a reasonable fit without fixing some parameters to a value given by our 1D analysis. We assigned the letter $a$ to fits where all the parameters are free to vary during the fitting process. If only geometrical parameters such as PA or $\epsilon$ are kept fixed then we assigned a $b$, and whenever we also needed to fix size-related quantities we considered them as a $c$. This quality check classification scheme for each galaxy was performed by at least two reviewers. In the case of disagreement, another reviewer checked the quality flag and provided the final classification. The number of galaxies with each particular flag is shown in Table 7 .
Table 7. Results from the visual quality check of the photometric decompositions.

\begin{tabular}{ccc}
\hline \hline $\begin{array}{c}\text { Flag number } \\
(1)\end{array}$ & $\begin{array}{c}\text { Flag letter } \\
(2)\end{array}$ & $\begin{array}{c}\text { Number } \\
(3)\end{array}$ \\
\hline \multirow{2}{*}{1} & $a$ & 190 \\
& $b$ & 14 \\
& $c$ & 122 \\
\hline \multirow{2}{*}{2} & $a$ & 22 \\
& $b$ & 5 \\
& $c$ & 26 \\
\hline 3 & $a$ & 13 \\
& $b$ & 0 \\
& $c$ & 10 \\
\hline & $a$ & 1 \\
& $b$ & 0 \\
& $c$ & 1 \\
\hline
\end{tabular}

Notes. (1) Different numbers represent galaxies with both good imaging and no other strong extra components (spiral arms or rings) affecting the fit (flag 1), good imaging but strong extra components that can influence the final fit (flag 2), poor imaging but no strong extra components affecting the final fit (flag 3 ) and poor imaging and strong extra components (flag 4); (2) Different letters represent whether or not: $(a)$ all the parameters are allowed to vary during the fitting process; $(b)$ only geometrical parameters such as PA or $\epsilon$ are kept fixed; and (c) size-related quantities are also fixed.

Only $\sim 6 \%$ of the images were classified as poor quality and $\sim 14 \%$ present strong extra components that might significantly affect the fit. On the other hand, $58 \%$ of the fits were performed allowing all the parameters to vary during the fit. This highlights the high level of convergency in our fits since $\sim 73 \%$ of our models require the constraint of $>10$ parameters. Regarding those fits with fixed quantities, only $5 \%$ of the fits required the ellipticities or PA of any of the components to be fixed to the values obtained from the 1D radial profiles. However, $\sim 37 \%$ of the fits have been performed fixing a size-related quantity of the model. We find that in approximately half of these cases the fixed parameter was the break radius of the disc in the Type II and Type III profiles. 
J. Méndez-Abreu et al.: Two-dimensional multi-component photometric decomposition of CALIFA galaxies

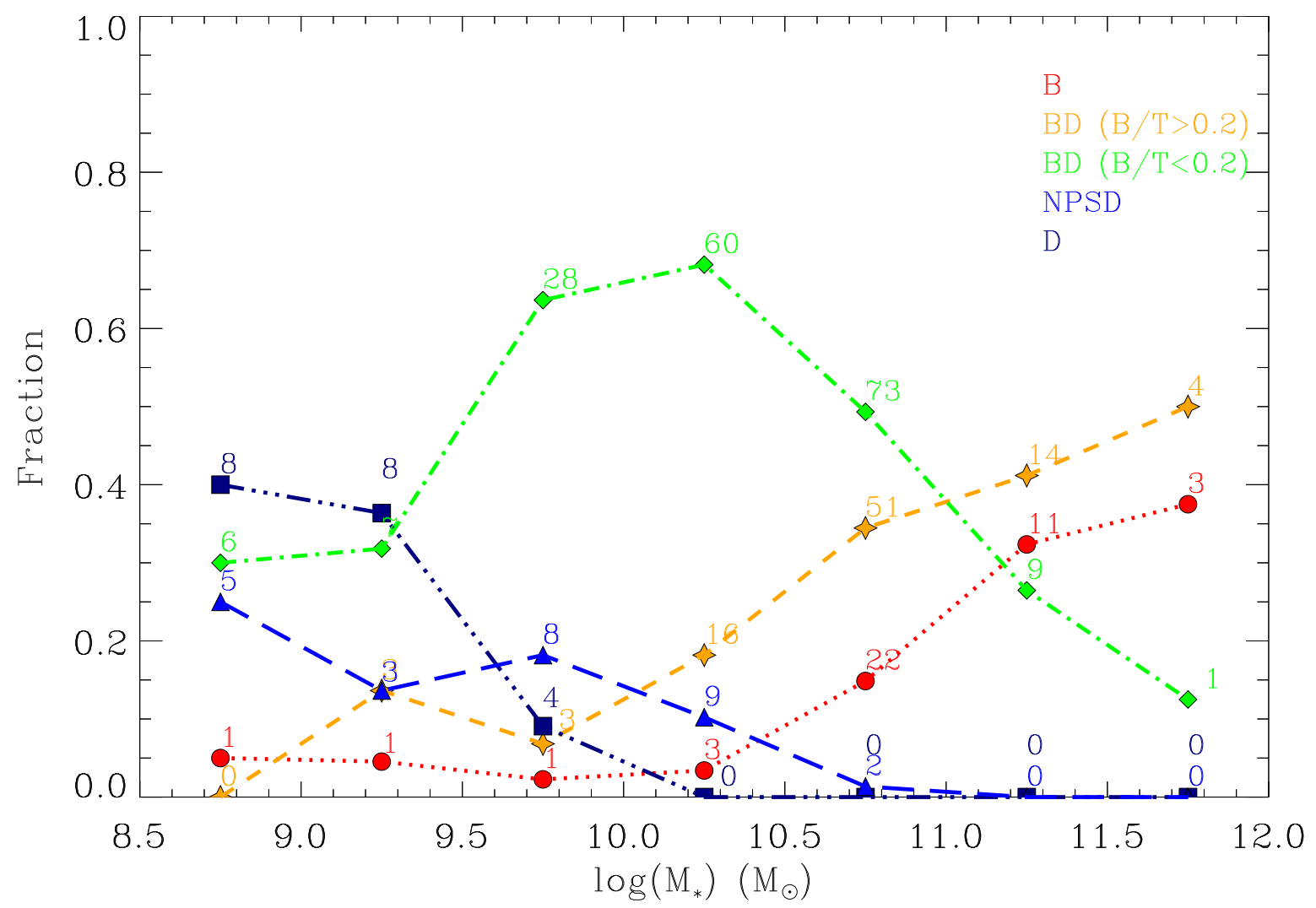

Fig. 9. Fraction of the final models used in the photometric decomposition as a function of stellar mass. Red circles represent single Sérsic models (B). Orange stars show models composed of a bulge+disc (BD) with $\mathrm{B} / \mathrm{T}>0.2$. Green diamonds display models composed of a bulge+disc with with $\mathrm{B} / \mathrm{T}<0.2$. Navy blue squares show models with no bulge but pure disc (D). Blue triangles represent models with a NPS+disc (NPSD). The five different combination of structures include broken profiles and/or bars. The number of galaxies in each bin is also shown.

As explained in Sect.4.3.2, constraining the parameters of disc breaks in a multi-component decomposition is not straightforward, but it is the only way to consistently compare with the other galaxy structures. It is worth noticing that the fixing of the break radius does not affect our classification nor the results shown in Sect.4.3.2.

\subsection{Statistical errors based on mock galaxy simulations}

The formal errors obtained from the minimisation procedure are usually not representative of the real errors on the fitted parameters (Méndez-Abreu et al. 2008). This is mainly because possible covariance terms are neglected in the error computation process. Therefore, to provide our structural parameters with a robust error estimate we carry out different tests applying Monte Carlo techniques to mock galaxies. This procedure allows us to improve both the accuracy of our error estimates, avoiding the otherwise necessary exploration of the full parameter space to account for the parameters' covariance, and to determine the observational limits of our photometric decomposition.

We devised a set of tailor-made simulations for each combination of structures fitted to our sample galaxies (see Table 3). For each set, we simulate 500 mock galaxies with structural parameters constrained within the limits of our real galaxy sample. For the sake of simplicity we assumed the $i$-band parameters as representative of our galaxies. Altogether we have created 7000 mock galaxies that are built in the following way. First, the total apparent magnitude of the galaxy is randomly selected within the observed range $m_{i}=[11,14]$. Then, the values of the relative luminosity ratios of the bulge $(\mathrm{B} / \mathrm{T})$, disc $(\mathrm{D} / \mathrm{T})$, bar (Bar/T), and NPS (NPS/T) are set according to the observed values for each combination of structures. The distribution of the luminosity ratios also matches that of the real galaxies, and a given luminosity is associated to each structure. The interval ranges for the size and shape parameters for each structure covered by our simulations are: $r_{\mathrm{e}}=\left[0.9^{\prime \prime}, 14^{\prime \prime}\right], n=[0.5,5], h=\left[4^{\prime \prime}\right.$, $\left.25^{\prime \prime}\right], r_{\text {break }}=\left[16^{\prime \prime}, 50^{\prime \prime}\right], h_{\text {out }}=\left[4^{\prime \prime}, 25^{\prime \prime}\right], r_{\text {bar }}=\left[5^{\prime \prime}, 30^{\prime \prime}\right]$. Once the mock galaxy structure is defined, the values of the surface brightness for the bulge $\left(\mu_{\mathrm{e}}\right)$, disc $\left(\mu_{0}\right)$, bar $\left(\mu_{0, \text { bar }}\right)$, and NPS $\left(\mu_{0, \mathrm{NPS}}\right)$ are derived using the equations to compute the total luminosity for each analytical representation of the galaxy structure. Finally, the axis ratio of the bulge $\left(q_{\text {bulge }}\right)$, disc $\left(q_{\text {disc }}\right)$ and bar $\left(q_{\mathrm{bar}}\right)$ components as well as the value of the position angle of the bulge $\left(\mathrm{PA}_{\text {bulge }}\right)$, disc $\left(\mathrm{PA}_{\text {disc }}\right)$ and bar $\left(\mathrm{PA}_{\mathrm{bar}}\right)$ are generated from a random uniform distribution, where no constraints are adopted. In the particular case of the double barred galaxies the typical values for the mock galaxy generation were obtained from de Lorenzo-Cáceres et al. (in prep.).

Mock galaxies are placed at a distance of $67 \mathrm{Mpc}$ that corresponds to the median value of our real sample. The galaxy models are then convolved with the mean PSF of the $i$-band SDSS images (see Sect. 3.3) to reproduce the typical spatial resolution. In addition, we adopt the pixel scale $(0.396 \mathrm{arcsec} / \mathrm{px})$, and the typical values of the CCD gain (4.86 $\left.\mathrm{e}^{-} / \mathrm{ADU}\right)$ and read-out noise $\left(5.76 \mathrm{e}^{-}\right)$to mimic the instrumental setup of the SDSS data. Finally, a background and photon noise are added to the artificial images to yield a signal-to-noise ratio to match the observed one.

The mock galaxies are then fitted using GASP2D as if they were real galaxies. The comparison between the input and output values of the fitted parameters is used to compute the errors. 


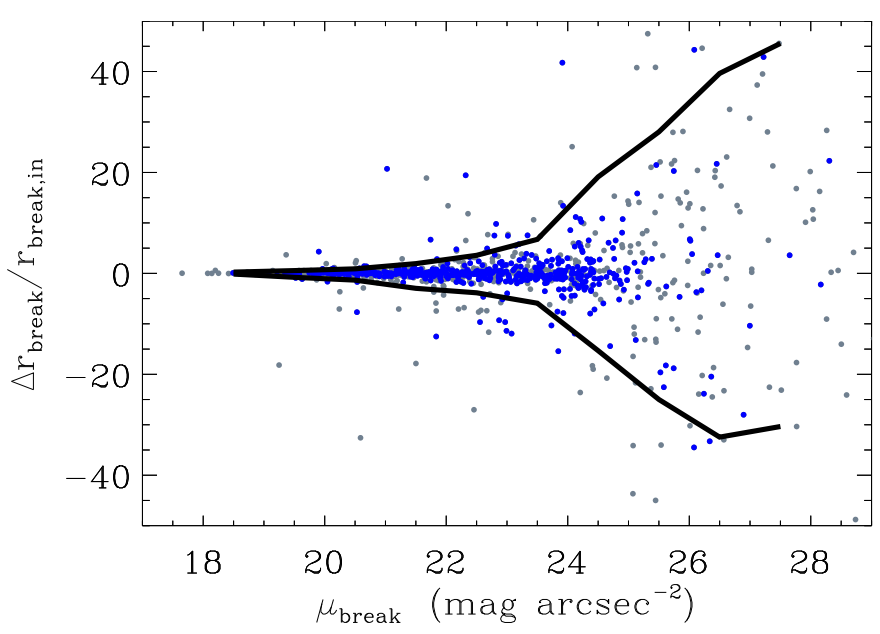

Fig. 10. Relative percentage error in the measurement of the break radius $\left(\left(r_{\text {break,in }}-r_{\text {break,out }}\right) / r_{\text {break,in }}\right)$ as a function of the surface brightness at $r_{\text {break }}$. The individual points represent all the mock galaxies with a broken disc independently of the presence of other components. Type II and Type III disc profiles are shown with grey and blue circles, respectively. The thick lines enclose $90 \%$ of the galaxies.

Mock galaxies were split into three different bins of magnitude $\left(11<m_{i}<12,12<m_{i}<13,13<m_{i}<14\right)$. In each bin, the mean and the standard deviation of the relative errors are adopted as the systematic and statistical errors for the observed galaxies for the parameters $\left(\mu_{\mathrm{e}}, r_{\mathrm{e}}, n, \mu_{0}, h, h_{\text {out }}, r_{\text {break }}, \mu_{0, \mathrm{bar}}, a_{\mathrm{bar}}\right.$, $\left.\mu_{0, \mathrm{NPS}}\right)$, whereas the mean and standard deviation of the absolute errors are adopted as the systematic and statistical errors for the observed galaxies for the set $\left(q_{\text {bulge }}, \mathrm{PA}_{\text {bulge }}, q_{\text {disc }}, \mathrm{PA}_{\text {disc }}, q_{\mathrm{bar}}\right.$, $\left.\mathrm{PA}_{\mathrm{bar}}\right)$. Since the systematic errors are generally smaller than the statistical errors they were added in quadrature. Errors are then assigned to each galaxy depending on its apparent magnitude. The final errors for each parameter are included in Table 1.

\section{Limitations to the measurement of breaks in disc galaxies}

The mock galaxy simulations described in the previous section also allow us to quantify the limitations for a robust measurement of disc breaks. To this end, we combined the six sets of simulations involving the presence of a broken disc independently of the presence of other components (see Table 3). The final sample includes 3000 mock galaxies. Figure 10 shows the relative error in the measurement of the break radius as a function of the surface brightness where the break occurs. It is worth noting that both Type II and Type III profiles are represented in the simulations and they are shown with grey and blue circles, respectively. We find that disc breaks can be robustly measured up to a SB of $24 \mathrm{mag} / \operatorname{arcsec}^{2}$ (within a $\sigma \sim 3 \%$ ). However, beyond this limit the determination of the break radius is more uncertain becoming completely undefined for $\mu_{\text {break }}>25 \mathrm{mag} / \operatorname{arcsec}^{2}$. These simulations demonstrate the limits of the SDSS imaging in accurately measuring breaks in disc galaxies.

\subsection{Comparison with previous CALIFA data}

A simple approach to understanding the structure of galaxies is the growth curve analysis. Walcher et al. (2014) analysed the CALIFA mother sample using this methodology on SDSS-DR7 images. They derived reliable values of the total magnitude, halflight major axis, axis ratios, and position angles of the CALIFA galaxies. These measurements have been used in several works within the CALIFA collaboration and therefore it is instructive to compare them with the results of this paper.

Since growth curve analysis does not separate different structural components but considers the galaxy a single entity, a meaningful comparison can only be achieved for our galaxies classified as single Sérsic models (B). Figure 11 shows the values of the effective radius $\left(r_{\mathrm{e}}\right)$, axis ratio $(q)$ and position angle (PA) obtained through the growth curve and photometric decomposition analysis. Different methods show very good agreement in terms of the geometrical parameters, with no systematic variations and an absolute scatter of 0.03 and 4 degrees in $q$ and PA, respectively. On the contrary, the values of $r_{\mathrm{e}}$ do show a systematic offset, with shorter values measured using the growth curve analysis. This result is expected since the Sérsic parameterisation assumes the galaxy model is extended to infinity, and therefore part of the galaxy light is still present in the outer and low surface brightness wings of the profile. In Fig. 11 we have labelled those galaxies with a Sérsic index $n>5.5$ in red. We show how the larger deviations actually appear in these galaxies where the profile wings are more prominent. The relative scatter between the two methods is $\sim 20 \%$.

\subsection{Comparison with the literature}

Given the human-supervised nature of the photometric decompositions presented here, the choice of the number of components included in the fit is a relevant source of uncertainty which is difficult to account for. Two-component (bulge+disc) models are the usual approach to deal with large datasets in an automatic way. In these cases the error bars associated with certain parameters might be excessively large due to the absence of critical structures such as bars (e.g. Gadotti 2008). Best suited multicomponent fits alleviate this problem but introduce the human factor. In this section we present a comparison of our results against two recent works comprising large datasets: Simard et al. (2011), who apply an automatic up-to-two-component fitting procedure to the SDSS-DR7, and Salo et al. (2015), who perform human-supervised multi-component fits of the whole $\mathrm{S}^{4} \mathrm{G}$ sample (Sheth et al. 2010). The main results are shown in Figs. 12 and 13, respectively.

Simard et al. (2011) analyse the $g$-and $r$-band images of over a million galaxies retrieved from the SDSS-DR7. They apply the GIM2D code (Simard 1998) to perform automatic one- (single Sérsic) and two-component (bulge+disc) decompositions of the whole sample, by using the information from the two bands simultaneously. In Fig. 12 we plot some relevant bulge/elliptical and disc parameters as obtained from both their approach and our multi-component fitting procedure in $r$-band. The number of components is consistently taken into account, so the galaxies that we classify as ellipticals (see Sect. 4.2) are compared with the one-component fit from Simard et al. (2011). Otherwise, the bulge+disc decomposition is considered. Both options are plotted for the cases with B/BD morphologies (see Sect. 4.2). It is worth noting that Simard et al. (2011) also provide twocomponent fits in which the Sérsic index is fixed to $n=4$. Those fits are not used in this comparison.

There are 25 galaxies in common between our sample and Simard et al. (2011). Galactic location on the sky explains the low number of common galaxies, as many CALIFA galaxies belong to sky regions not included in the SDSS Legacy Survey used by Simard et al. (2011). The large dispersion shown in the plots is expected as Simard et al. (2011) do not include other relevant structures such as bars, inner discs, or main disc truncations in their fits. In general, the bulge parameters $\left(n\right.$ and $\left.r_{\mathrm{e}}\right)$ measured 
J. Méndez-Abreu et al.: Two-dimensional multi-component photometric decomposition of CALIFA galaxies
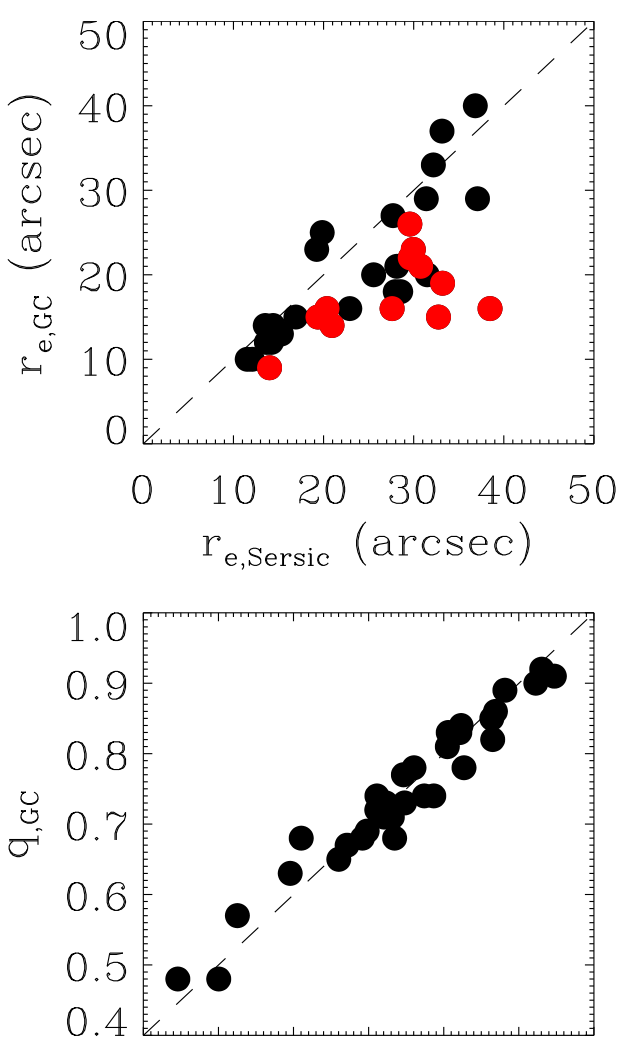

0.40 .50 .60 .70 .80 .91 .0

q,Sersic

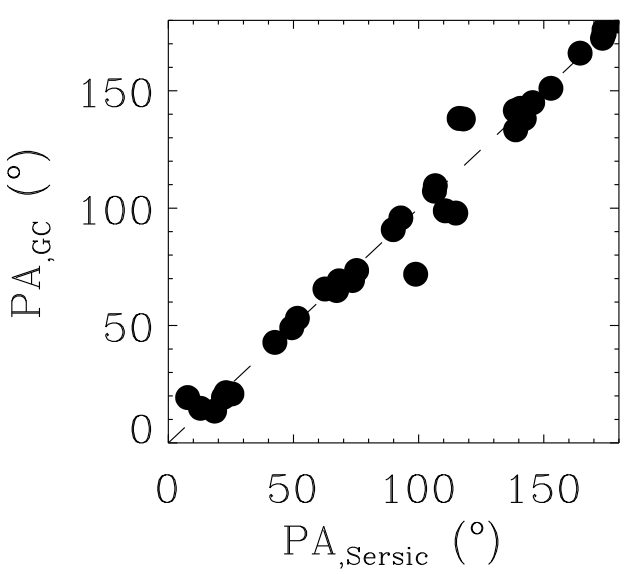

Fig. 11. Comparison of our photometric decomposition $r$-band estimated parameters (labelled as Sérsic) with those obtained from the growth curves (labelled as GC) in Walcher et al. (2014). Only those galaxies fitted with a single Sérsic profile have been used in this comparison. From top to bottom, panels show the galaxy effective radius, axis ratio, and position angle, respectively. Red points in the upper panel represent galaxies fitted with a single Sérsic with $n>5.5$.

by Simard et al. (2011) tend to be larger than those from this work, as their bulges need to account for other central structures as well.

The $S^{4} \mathrm{G}$ sample consists of 2352 galaxies observed with the Spitzer Infrared Array Camera (IRAC) in the near-infrared 3.6 and $4.5 \mu \mathrm{m}$ bands. GALFIT (Peng et al. 2002) multi-component photometric decompositions for all the $3.6 \mu \mathrm{m}$ images are presented in Salo et al. (2015). The strategy followed in their analysis is analogous to the one shown in this paper. There are notwithstanding some important differences that are worth
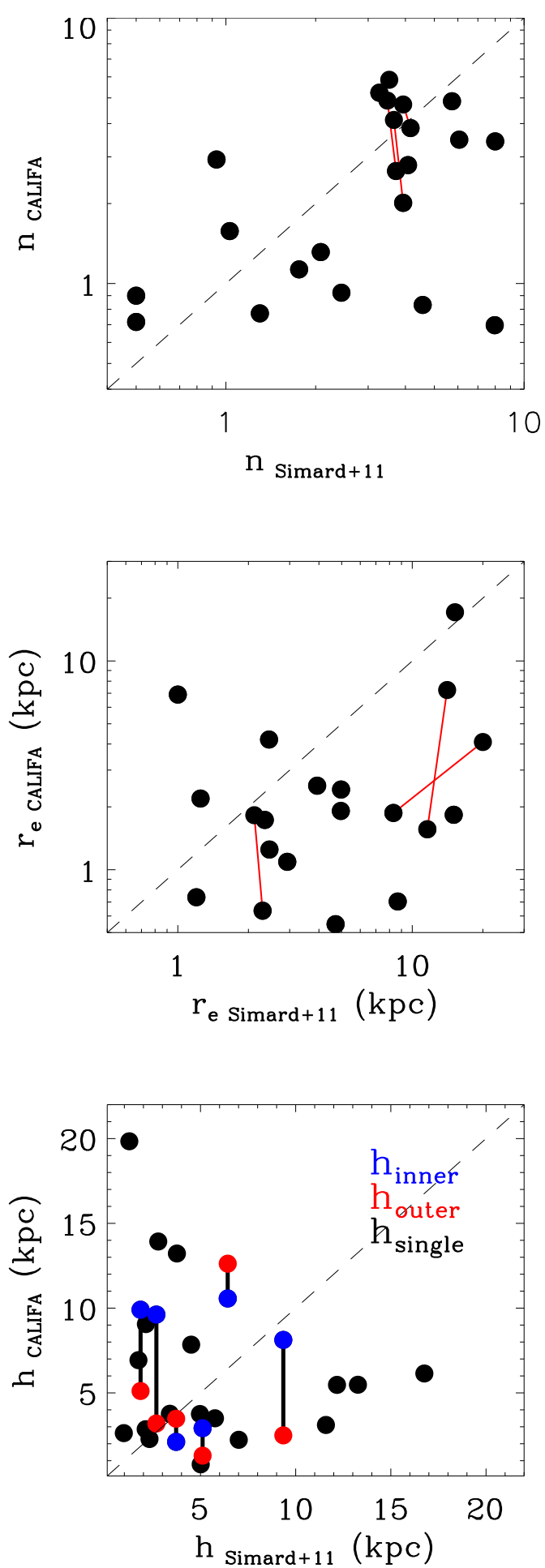

Fig. 12. Comparison of our $r$-band estimated parameters with those from the GIM2D photometric decompositions from Simard et al. (2011). Top and middle panels show bulge Sérsic index and effective radius respectively. Parameters from the one- and two-component fits for the early-type galaxies with $\mathrm{B} / \mathrm{BD}$ morphology are linked with solid red lines. The bottom panel compares the disc scale lengths. Inner and outer disc scale lengths are distinguished in the case of breaks as blue and red dots, respectively, and linked with a solid black line. Simard et al. (2011) do not take into account breaks in their analysis.

pointing out. First, Salo et al. (2015) do not use truncated discs in their models; instead only single exponential discs are fitted. If there are clear differences between the inner and outer disc 

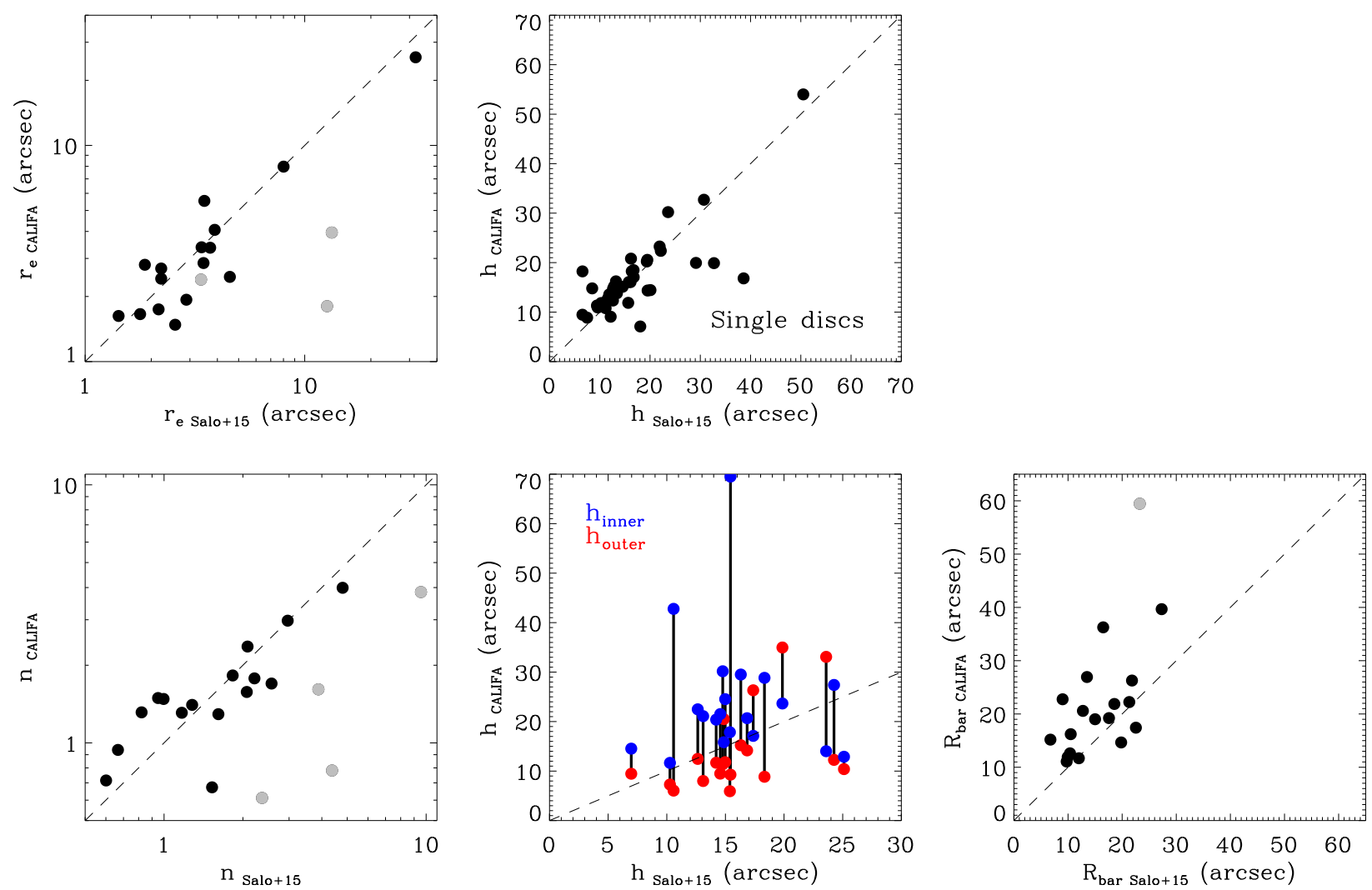

Fig. 13. Comparison of our $r$-band best-fit parameters with those from the multi-component photometric decompositions of the $3.6 \mu \mathrm{m}$ images of the $\mathrm{S}^{4} \mathrm{G}$ galaxies (Salo et al. 2015). There are 65 galaxies in common between our sample and $\mathrm{S}^{4} \mathrm{G}$. Left panels correspond to elliptical/bulge parameters: effective radius (top) and Sérsic index (bottom). Controversial bulges (see text) are plotted as grey dots. Middle panels compare the disc scale lengths for single discs (top) and truncated discs as classified by this work (bottom). Salo et al. (2015) only include single discs in their decompositions. Inner and outer disc scale lengths are distinguished in the case of breaks as blue and red dots, respectively. For the sake of clarity, the 1:1 axis proportion in the bottom panel has not been kept. The right panel shows the comparison of the bar lengths for the 19 galaxies classified as barred by both works. The use of two different disc components (an exponential and Ferrers) in the $\mathrm{S}^{4} \mathrm{G}$ fit explain the difference for NGC 5957 (grey dot).

regions, they include an extra component that it is added up to the others. This component is tagged as a disc but can have a Ferrers or Sérsic profile if that better resembles the image. $S^{4} \mathrm{G}$ galaxies can therefore be classified as having multiple disc structures when only one is actually present. In other cases however, the galaxy can have two actual discs (e.g. the usual disc plus an inner disc). Secondly, for very inclined galaxies, Salo et al. (2015) fit three-dimensional discs. Whereas the usual exponential disc is considered infinitesimally thin, they include "edgediscs" with vertical extension for those galaxies with $q \lesssim 0.2$. Since we apply a threshold in inclination, none of the CALIFA galaxies in common with $S^{4} \mathrm{G}$ have been fitted with an edgedisc.

The human-supervised choices of components from Salo et al. (2015) and this work match exactly for 47 out of the 65 galaxies common to both samples. For the reasons stated above, we consider multiple disc structures in the $S^{4} \mathrm{G}$ classifications as equivalent to one disc component in the CALIFA galaxies. Similarly, NPS and bulge components are equally taken into account. As indicated in Sect. 3.1, and also discussed in Salo et al. (2015), NPSs are usually unresolved bulges. For these cases, it is always the $S^{4} G$ classification that includes a NPS, while a proper bulge is shown in the CALIFA analysis, as expected due to the lower resolution of the near-infrared images when compared to SDSS data.

The fact that the classifications from both teams, which use different codes and images, match for $72 \%$ of the common sample, is an indication of the robustness of the human-supervised multi-component photometric decomposition techniques. The 19 non-matching galaxies show relevant differences in the classifications, such as the presence of bulges or bars. In particular, we include bars in five CALIFA galaxies which are unbarred for the $S^{4} \mathrm{G}$ team, whereas the opposite occurs for seven galaxies. Since it has been found that the measured bar fraction increases in near-infrared observations (e.g. Marinova \& Jogee 2007), a higher bar occurrence in the $S^{4} G$ data is expected. No significant differences are found in the common sample of 65 galaxies. Regarding the early-types, there is one CALIFA elliptical galaxy classified as S0 by $\mathrm{S}^{4} \mathrm{G}$ and viceversa.

Figure 13 shows the comparison of bulge (or single Sérsic), disc, and bar parameters between our $r$-band images and the $3.6 \mu \mathrm{m}$ images used by Salo et al. (2015). Overall, the results are in good agreement. We have visually explored the outliers shown in the plots for the bulge parameters (Sérsic index and effective radius). Some correspond to bulges that acquire high SB values in the outer galaxy regions, even surpassing the disc profile in the Salo et al. (2015) fits. This is considered unrealistic in our decomposition and therefore they show too high Sérsic index and effective radius values with respect to the results from this work. In other cases, different bar fits or even the absence of a bar for one of the galaxies accounts for inconsistencies in bulge parameters.

Twenty galaxies of the common sample host truncated discs, as classified in this work. Salo et al. (2015) do not include breaks and truncations in their decomposition. Truncations can 
be related to structures such as rings or bars and it is therefore not obvious which disc region, inner or outer, is best suited for the comparison with a single disc such as the one provided for the $\mathrm{S}^{4} \mathrm{G}$ galaxies. Moreover, the estimated properties of a single disc may also be affected by other structures or even image parameters such as sky background, thus are biased towards inner or outer measurements depending on each case. Salo et al. (2015) show that their single discs are a good estimate of a "mean" disc of the inner and outer values. They also find that outer discs in Type II galaxies better resemble the properties of actual single discs. In Fig. 13 we compare both the inner and outer disc scale lengths derived in this work with the single $S^{4} G$ scale lengths. Values from the outer disc regions lie slightly closer to the unity line, although the situation is different for each galaxy. Results for galaxies hosting single discs as concluded by both CALIFA and $\mathrm{S}^{4} \mathrm{G}$ classifications are in full agreement.

There are 19 galaxies classified as barred by both Salo et al. (2015) and this work (bottom right panel of Fig. 13). The main outlier corresponds to NGC 5957. Salo et al. (2015) fit a bulge, bar, and two discs to this galaxy. While the main disc has an exponential profile, the second one is fitted with a Ferrers profile. In this work NGC 5957 is fitted with a bulge, bar, and single disc. We have verified that our bar corresponds to their Ferrers disc, and thus the inconsistency in the results, while we do not find signatures of any other inner bar in the optical images.

\section{Conclusions}

This paper presents the multi-component photometric decomposition of 404 galaxies from the CALIFA DR3 survey. Our aim is to provide the community with an accurate photometric characterisation of the multiple stellar structures shaping the CALIFA galaxies; namely bulges, bars, and discs.

The galaxy sample covers all galaxies included in the CALIFA final data release, both from the mother sample and the extension sample, except those in mergers, interactions, and those that are highly inclined. We adopted a human-supervised strategy where up to three structural components: bulge/NPS (nuclear point source), disc (including breaks), and bar are used to provide the best fit for each galaxy. The final combination of structures was individually determined by the code-user after checking several possibilities. We have thoroughly compared our results with previous works from the literature (Simard et al. 2011; Salo et al. 2015) obtaining a reasonable agreement when the different galaxy components are carefully selected, but different results when automatic methods are used. We consider this as an indication of the robustness of the human-supervised multicomponent photometric decomposition techniques. Counting disc breaks as different structures, we used 13 different combinations of structures to describe our sample galaxies.

We focused on the incidence of the different structures in our sample. Since the galaxies extracted from the mother sample are volume-correctable, we have studied the frequency of the different structures for the whole observed sample and volume -corrected. Our main conclusions are:

- We found an average bar fraction in our volume-corrected sample of $57 \%$, which is consistent with previous results in the literature. The volume-corrected bar fraction shows a drop toward late type galaxies; unfortunately, the number of galaxies in the late-type bin is too small to draw statistical conclusions. The observed bar fraction (using the whole sample) is relatively constant with Hubble type. Regarding the mass dependance of the bar fraction, the volume-corrected bar fraction drops from $\sim 75 \%$ at $M_{\star}=$ $10^{9.5} M_{\odot}$ to $\sim 25 \%$ at $M_{\star}=10^{11} M_{\odot}$.

- We explored the frequency of different disc types by using 2D surface-brightness models including broken exponential profiles and found that $62 \%, 28 \%$, and $10 \%$ of our volume corrected disc sample are better represented with a Type I (pure exponential), a Type II (down-bending), and a Type III (up-bending) profile, respectively. These fractions are in strong disagreement with previous results in the literature (Erwin et al. 2008; Gutiérrez et al. 2011). We argue that the different methodologies are the main explanation for these differences. In our 2D analysis we are simultaneously fitting all different galaxy components whereas 1D studies usually fit only piecewise exponentials to pre-defined regions of the SB profile. Despite the quantitative differences, we found the same trends observed in the previous works, that is, a decrease in the fraction of Type I profiles with Hubble type (from Sa to Sc), an increase for the Type II profiles and an almost constant fraction for Type III galaxies. No significant trends are found in terms of the stellar mass or the presence of bars.

- We also studied the incidence of pure discs and/or small unresolved bulges in our sample. Regarding the presence of a bulge and its prominence, we find a clear segregation of the structural composition of galaxies according to stellar mass. At high masses $\left(\log \left(M_{\star} / M_{\odot}\right)>11\right)$, galaxies modelled with a single Sérsic or a bulge+disc with $\mathrm{B} / \mathrm{T}>0.2$ represent the dominant population. At intermediate masses $(9.5<$ $\left.\log \left(M_{\star} / M_{\odot}\right)<11\right)$, galaxies described with bulge+disc but $\mathrm{B} / \mathrm{T}<0.2$ are preponderant whereas in the low mass end $\left(\log \left(M_{\star} / M_{\odot}\right)<9.5\right)$, the prevailing population is constituted by galaxies modelled with either pure discs or nuclear point sources+discs (i.e. no discernible bulge). This trend of the fitted model with galaxy mass is also consistent with the trend of the $\mathrm{B} / \mathrm{T}$ ratio with both Hubble type and mass. We found a clear decrease of $\mathrm{B} / \mathrm{T}$ with both increasing Hubble type and decreasing mass with an average volume corrected $\mathrm{B} / \mathrm{T}$ value of 0.14 .

The work presented in this paper focuses on describing the photometric decomposition pipeline and the incidence of the different structural components in the final CALIFA data release. It also, however, sets the basis for new studies combining photometric information with the wealth of 2D spatially resolved spectroscopic information provided by the CALIFA survey.

Acknowledgements. J.M.A. and V.W. acknowledges support from the European Research Council Starting Grant (SEDmorph; P.I. V. Wild). L.S.M acknowledges financial support from the Spanish Ministerio de Economía y Competitividad (MINECO) via grant AYA2012-31935. A.d.L.C. acknowledges support from the UK Science and Technology Facilities Council (STFC) grant ST/J001651/1 and from the Spanish Ministry of Economy and Competitiveness (MINECO) grant AYA2011-24728. C.C.-T. thanks the support of the Spanish Ministerio de Educación, Cultura y Deporte by means of the FPU fellowship program and the support from the Plan Nacional de Investigación y Desarrollo funding programs, AYA2012-30717 and AyA2013-46724P, of Spanish Ministerio de Economía y Competitividad (MINECO)'. E.F. acknowledges support by the MINECO grant AYA2014-53506-P and the Junta de Andalucía grant FQM108. J.L.A. acknowledges financial support from the MINECO grant AYA2013-43188-P. E.M.C. and L.C. are supported by Padua University through grants 60A02-5857/13, 60A02$5833 / 14$, and 60A02-4434/15. L.C. acknowledges the University of St. Andrews for the hospitality while this paper was in progress. L.G. was supported in part by FONDECYT through grant 3140566 and the US National Science Foundation under Grant AST-1311862. I.M. acknowledges financial support from the MINECO grant AYA-42227-P. RAOM acknowledges support from CAPES (Brazil) through a PDJ fellowship from project 88881.030413/2013-01, program CSF-PVE. P.P. is supported by FCT through the Investigador FCT Contract No. IF/01220/2013 and POPH/FSE (EC) by FEDER funding through the program COMPETE. P.S.B. acknowledge financial support from the: CONICYT-Chile 
Basal-CATA PFB-06/2007 and the AYA2013-48226-C3-1-P by the Ministerio de Ciencia e Innovacion. This paper is based on data from the Calar Alto Legacy Integral Field Area Survey, CALIFA (http://califa.caha.es), funded by the Spanish Ministery of Science under grant ICTS-2009-10, and the Centro Astronómico Hispano-Alemán. Based on observations collected at the Centro Astronómico Hispano Alemán (CAHA) at Calar Alto, operated jointly by the Max-Planck Institut für Astronomie and the Instituto de Astrofísica de Andalucía (CSIC). We acknowledge the use of SDSS data (http://www.sdss. org/collaboration/citing-sdss/).

\section{References}

Abadi, M. G., Navarro, J. F., Steinmetz, M., \& Eke, V. R. 2003, ApJ, 591, 499 Abazajian, K. N., Adelman-McCarthy, J. K., Agüeros, M. A., et al. 2009, ApJS, 182,543

Aguerri, J. A. L. 2012, Adv. Astron., 2012, 382674

Aguerri, J. A. L., \& Trujillo, I. 2002, MNRAS, 333, 633

Aguerri, J. A. L., Elias-Rosa, N., Corsini, E. M., \& Muñoz-Tuñón, C. 2005, A\&A, 434, 109

Aguerri, J. A. L., Méndez-Abreu, J., \& Corsini, E. M. 2009, A\&A, 495, 491

Akaike, H. 1974, IEEE Transactions on Automatic Control, 716

Allen, P. D., Driver, S. P., Graham, A. W., et al. 2006, MNRAS, 371, 2

Athanassoula, E., Machado, R. E. G., \& Rodionov, S. A. 2013, MNRAS, 429, 1949

Azzollini, R., Trujillo, I., \& Beckman, J. E. 2008, ApJ, 684, 1026

Balcells, M., Graham, A. W., \& Peletier, R. F. 2007, ApJ, 665, 1084

Barazza, F. D., Jogee, S., \& Marinova, I. 2008, ApJ, 675, 1194

Benítez, E., Méndez-Abreu, J., Fuentes-Carrera, I., et al. 2013, ApJ, 763, 136

Bertin, E., \& Arnouts, S. 1996, A\&AS, 117, 393

Bland-Hawthorn, J., Vlajić, M., Freeman, K. C., \& Draine, B. T. 2005, ApJ, 629, 239

Blanton, M. R., Hogg, D. W., Bahcall, N. A., et al. 2003, ApJ, 592, 819

Böker, T., Laine, S., van der Marel, R. P., et al. 2002, AJ, 123, 1389

Boroson, T. 1981, ApJS, 46, 177

Brooks, A., \& Christensen, C. 2016, Galactic Bulges, 418, 317

Buta, R. J., Sheth, K., Athanassoula, E., et al. 2015, ApJS, 217, 32

Byun, Y. I., \& Freeman, K. C. 1995, ApJ, 448, 563

Caon, N., Capaccioli, M., \& D’Onofrio, M. 1993, MNRAS, 265, 1013

Corsini, E. M., Aguerri, J. A. L., Debattista, V. P., et al. 2007, ApJ, 659, L121

de Lorenzo-Cáceres, A., Vazdekis, A., Aguerri, J. A. L., Corsini, E. M., \& Debattista, V. P. 2012, MNRAS, 420, 1092

de Lorenzo-Cáceres, A., Falcón-Barroso, J., \& Vazdekis, A. 2013, MNRAS, 431, 2397

de Souza, R. E., Gadotti, D. A., \& dos Anjos, S. 2004, ApJS, 153, 411

Emsellem, E., Cappellari, M., Krajnović, D., et al. 2011, MNRAS, 414, 888

Erwin, P. 2004, A\&A, 415, 941

Erwin, P. 2015, ApJ, 799, 226

Erwin, P., \& Sparke, L. S. 2002, AJ, 124, 65

Erwin, P., Beckman, J. E., \& Pohlen, M. 2005, ApJ, 626, L81

Erwin, P., Pohlen, M., \& Beckman, J. E. 2008, AJ, 135, 20

Ferrers, N. M. 1877, Quart. J. Pure and Appl. Math, 14, 1

Freeman, K. C. 1970 , ApJ, 160, 811

Gadotti, D. A. 2008, MNRAS, 384, 420

Gadotti, D. A. 2009, MNRAS, 393, 1531

García-Benito, R., Zibetti, S., Sánchez, S. F., et al. 2015, A\&A, 576, A135

Gomes, J. M., Papaderos, P., Vílchez, J. M., et al. 2016, A\&A, 585, A92

Governato, F., Mayer, L., Wadsley, J., et al. 2004, ApJ, 607, 688

Gutiérrez, L., Erwin, P., Aladro, R., \& Beckman, J. E. 2011, AJ, 142, 145

Häußler, B., Bamford, S. P., Vika, M., et al. 2013, MNRAS, 430, 330

Head, J. T. C. G., Lucey, J. R., Hudson, M. J., \& Smith, R. J. 2014, MNRAS, 440,1690

Hickson, P., Auman, J. R., Ninkov, Z., et al. 1982, ApJ, 258, 53

Hubble, E. P. 1926, ApJ, 64, 321

Huertas-Company, M., Aguerri, J. A. L., Bernardi, M., Mei, S., \& Sánchez Almeida, J. 2011, A\&A, 525, A157
Husemann, B., Jahnke, K., Sánchez, S. F., et al. 2013, A\&A, 549, A87

Hyde, J. B., \& Bernardi, M. 2009, MNRAS, 394, 1978

Kautsch, S. J. 2009, PASP, 121, 1297

Kautsch, S. J., Grebel, E. K., Barazza, F. D., \& Gallagher, J. S. III 2006, A\&A, 445,765

Kormendy, J. 1977, ApJ, 218, 333

Kormendy, J., Fisher, D. B., Cornell, M. E., \& Bender, R. 2009, ApJS, 182, 216 Lackner, C. N., \& Gunn, J. E. 2012, MNRAS, 421, 2277

Laine, S., Shlosman, I., Knapen, J. H., \& Peletier, R. F. 2002, ApJ, 567, 97

Laine, J., Laurikainen, E., Salo, H., et al. 2014, MNRAS, 441, 1992

Laurikainen, E., Salo, H., \& Buta, R. 2005, MNRAS, 362, 1319

Laurikainen, E., Salo, H., Buta, R., \& Knapen, J. H. 2007, MNRAS, 381, 401

Laurikainen, E., Salo, H., Buta, R., Knapen, J. H., \& Comerón, S. 2010, MNRAS, 405, 1089

Lee, G.-H., Park, C., Lee, M. G., \& Choi, Y.-Y. 2012, ApJ, 745, 125

Malin, D. F., \& Carter, D. 1980, Nature, 285, 643

Marino, R. A., Gil de Paz, A., Sánchez, S. F., et al. 2016, A\&A, 585, A47

Marinova, I., \& Jogee, S. 2007, ApJ, 659, 1176

Masters, K. L., Nichol, R. C., Hoyle, B., et al. 2011, MNRAS, 411, 2026

Masters, K. L., Nichol, R. C., Haynes, M. P., et al. 2012, MNRAS, 424, 2180

Méndez-Abreu, J., Aguerri, J. A. L., Corsini, E. M., \& Simonneau, E. 2008, A\&A, 478, 353

Méndez-Abreu, J., Sánchez-Janssen, R., \& Aguerri, J. A. L. 2010, ApJ, 711, L61

Méndez-Abreu, J., Sánchez-Janssen, R., Aguerri, J. A. L., Corsini, E. M., \& Zarattini, S. 2012, ApJ, 761, L6

Méndez-Abreu, J., Debattista, V. P., Corsini, E. M., \& Aguerri, J. A. L. 2014, A\&A, 572, A25

Méndez-Abreu, J., Aguerri, J. A. L., \& Falcon-Barroso, J. et al. (CALIFA collaboration) 2016, A\&A, submitted

Morelli, L., Halliday, C., Corsini, E. M., et al. 2004, MNRAS, 354, 753

Muñoz-Mateos, J. C., Sheth, K., Gil de Paz, A., et al. 2013, ApJ, 771, 59

Nair, P. B., \& Abraham, R. G. 2010, ApJ, 714, L260

Peebles, P. J. E., \& Nusser, A. 2010, Nature, 465, 565

Peng, C. Y., Ho, L. C., Impey, C. D., \& Rix, H. 2002, AJ, 124, 266

Peng, C. Y., Ho, L. C., Impey, C. D., \& Rix, H.-W. 2010, AJ, 139, 2097

Pérez, I. 2004, A\&A, 427, L17

Pohlen, M., \& Trujillo, I. 2006, A\&A, 454, 759

Prieto, M., Aguerri, J. A. L., Varela, A. M., \& Muñoz-Tuñón, C. 2001, A\&A, 367,405

Ruiz-Lara, T., Pérez, I., Florido, E., et al. 2016, MNRAS, 456, L35

Salo, H., Laurikainen, E., Laine, J., et al. 2015, ApJS, 219, 4

Sánchez, S. F., Kennicutt, R. C., Gil de Paz, A., et al. 2012, A\&A, 538, A8

Sánchez, S. F., Rosales-Ortega, F. F., Iglesias-Páramo, J., et al. 2014, A\&A, 563, A49

Sánchez, S. F., García-Benito, R., Zibetti, S., et al. 2016, A\&A, 594, A36

Sánchez-Blázquez, P., Rosales-Ortega, F. F., Méndez-Abreu, J., et al. 2014, A\&A, 570, A6

Sánchez-Menguiano, L., Sánchez, S. F., Pérez, I., et al. 2016, A\&A, 587, A70

Schwarz, G. E. 1978, Annals of Statistics, 6, 461

Send, U. 1982, A\&A, 112, 235

Sérsic, J. L. 1968, Atlas de galaxias australes (Cordoba, Argentina: Observatorio Astronomico)

Sheth, K., Regan, M., Hinz, J. L., et al. 2010, PASP, 122, 1397

Simard, L. 1998, in Astronomical Data Analysis Software and Systems VII, eds. R. Albrecht, R. N. Hook, \& H. A. Bushouse, ASP Conf. Ser., 145, 108

Simard, L., Mendel, J. T., Patton, D. R., Ellison, S. L., \& McConnachie, A. W. 2011, ApJS, 196, 11

Torres-Papaqui, J. P., Coziol, R., Andernach, H., et al. 2012, Rev. Mex. Astron. Astrofis., 48, 275

Trujillo, I., \& Pohlen, M. 2005, ApJ, 630, L17

Trujillo, I., Aguerri, J. A. L., Cepa, J., \& Gutiérrez, C. M. 2001a, MNRAS, 328, 977

Trujillo, I., Aguerri, J. A. L., Gutiérrez, C. M., \& Cepa, J. 2001b, AJ, 122, 38

Walcher, C. J., Wisotzki, L., Bekeraité, S., et al. 2014, A\&A, 569, A1

Weinzirl, T., Jogee, S., Khochfar, S., Burkert, A., \& Kormendy, J. 2009, ApJ, 696, 411 